Série des Documents de Travail

\author{
$n^{\circ}$ 2012-07 \\ Correlated Risks vs Contagion \\ in Stochastic Transition \\ Models \\ P. GAGLIARDINI ${ }^{1}$ \\ C. GOURIÉROUX ${ }^{2}$
}

First version : May, 2011

This version : March, 2012*

Les documents de travail ne reflètent pas la position du CREST et n'engagent que leurs auteurs. Working papers do not reflect the position of CREST but only the views of the authors.

\footnotetext{
1 University of Lugano.

2 CREST, CEPREMAP and University of Toronto (Canada).

* Acknowledgements : The first author gratefully acknowledges financial support of the Swiss National Science Foundation through the NCCR FINRISK network. The second author gratefully acknowledges financial support of NSERC Canada and the chair AXA/Risk Foundation : "Large Risks in Insurance".
} 


\title{
Correlated Risks vs Contagion in Stochastic Transition Models
}

\begin{abstract}
There is a growing literature on the possibility to identify correlation and contagion in qualitative risk analysis. Our paper considers this question by means of a model describing the joint dynamics of a set of individual binary processes. The two admissible values correspond to bad and good risk states of an individual. The risk correlation and its time dependence are captured by introducing a dynamic frailty, whereas the contagion passes through the effect of the lagged number of individuals in the bad risk state. We study carefully the dynamic properties of the joint process. Then, we focus on the limiting case of large populations (portfolios) and reconcile the microscopic and macroscopic dynamic views of the risk. The difficulty to identify in finite sample risk correlation and contagion is illustrated by means of Monte-Carlo simulations.
\end{abstract}

Keywords: Risk Dependence, Frailty, Systematic Risk, Contagion, Count Process, INAR Model, Compound Autoregressive Process, Affine Model, Credit Risk, Granularity Adjustment, Stochastic Intensity.

JEL classification: G12, C23. 


\section{Introduction}

The Law of Large Numbers is often invoked to justify the possibility to eliminate, or at least diminish, the risk of a portfolio by diversifying the type of investments. Typically, it was often considered that a large portfolio of individual life insurance contracts, or of mortgages, is almost riskfree, and might be priced by considering its expected value, called pure premium in Insurance. However, the possibility of diversification supposes satisfied the assumptions of the Law of Large Numbers, in particular the fact that the individual risks are not too dependent. The 2008-2011 financial crisis showed that the risk dependencies cannot be neglected and constitute a major component of the risk of the system when computing the reserves of a financial institution, or of an insurance company. The role of some dependencies was already highlighted in Basel 2 and Solvency 2 regulations [BCBS (2001), (2003)] beginning to be implemented before the crisis. These were the dependencies due to observable and unobservable common risk factors, called systematic factors, or frailties. For instance, the individual mortgages are often contracts with adjustable rates and monthly payments indexed on some prime rate. An increase of this prime rate implies an increase of the level of monthly payments for a lot of mortgages and, thus, correlated defaults. Similarly, the risk of life insurance contracts depends on the general uncertain increase of human lifetime, called longevity risk; this unobservable longevity risk becomes a major source of possible losses for a life insurance company.

After 2008, the regulation has been extended in two directions. First, it highlights contagion as a major source of risk dependencies. Second, it also focuses on the risk of the banking (financial) system as a whole, and not only on the separate analysis of the risks of the banks and insurance companies. The contagion effect is due to the interconnection between the banks and insurance companies by means of their debt and reinsurance structures. Typically, the failure of a company will imply losses for its lenders, and maybe the failure of some of them, then possibly failure of some lenders of the lenders, and so on. We get a propagation of the risk along a chain of failures.

It is clearly important to disentangle the two types of dependencies, namely the effects of shocks exogenous to the system and the contagion phenomenon, which is system endogenous, in order to measure their relative magnitude and to clarify their distinct roles. This distinction is crucial for instance for the computation of the reserves needed to protect the whole system, i.e., 
the reserves for systemic risk, and for the definition of the contribution of each financial institution to these reserves for systemic risk. There exist alternative approaches to identify these two components of risk dependencies, which are structural and reduced form approaches, respectively.

(i) The structural approach tries to get the precise knowledge of all possible connections between the institutions of interest. Typically, if we consider the European banking system, the idea is to know regularly the detailed balance sheets of all the banks, the proportion of debt of each bank with respect to each other ones, for each time to maturity and seniority level, etc. Then, we can construct the contagion network, and understand what might be the propagation in the system of an adverse shock. This is the principle of the so-called stress tests. However, this approach requires databases on the detailed balanced sheets, which exist for some countries, but are submitted to strict confidentiality restrictions. Moreover, the complexity of such a network increases very quickly with the number of institutions in the system. This explains why the first studies of this type focus on rather small networks of institutions [see e.g. Egloff, Leippold, Vanini (2007)], or countries with a sufficiently concentrated banking system [see e.g. Upper, Worms (2004) for Germany, or Gourieroux, Heam, Monfort (2011) for France]. But it seems difficult to apply this approach directly to US, for instance, due to the thousands of US financial institutions.

(ii) The reduced form approach tries to identify the two components of risk dependencies without the structural knowledge of the interconnection between the individual risks. The reduced form models for contagion consider specifications that capture how the defaults of some individuals influence the default intensities of the individuals, who are still alive. They are either (a) dynamic models written in continuous time, at the individual level ${ }^{1}$, and based on the notion of mutually exciting point processes introduced by Hawkes (1971) a,b, Hawkes, Oakes (1974), or (b) specifications based on the epidemic model introduced by Bailey (1953, 1957), Kendall (1956) [see the so-called infectious model used in a static framework by Davis, Lo (2001) a,b, Sakata, Isakado, Mori (2007), and its dynamic extension by Rulliere, Dorobantu, Cousin (2010)]. Specifications including both frailty and contagion effects are introduced e.g. in Frey, Backhaus (2003), Giesecke, Weber (2004), (2006), Azizpour, Giesecke (2008), a,b, and Lando, Nielsen (2010). For the analy-

\footnotetext{
${ }^{1}$ These models have to be distinguished from dynamic macroscopic models introduced to capture the volatility transmission [Gallo, Otranto (2007)], or the jump transmission [Ait-Sahalia, Cacho-Diaz, Laeven (2010)] between markets, even if they share common features with the microscopic models introduced for individual risks.
} 
sis of contagion and frailty effects, it is important to consider rather large portfolios of individual risks, which are sufficiently homogeneous with respect not only to their marginal risk distribution, but also to the two types of dependencies. Indeed, the Basel 2 regulation has already considered such benchmark models for the analysis of large portfolios of loans, mortgages, Credit Default Swaps when risk dependence arises from frailty effects only [see e.g. Schonbucher (2001), Frey, McNeil (2003)]. For such models, portfolio risk measures can be analytically approximated by their cross-sectional (i.e. large portfolio) limits [Vasicek (1987)], including first-order corrections called granularity adjustments [Gordy (2003), (2004), Gagliardini, Gourieroux, Monfort (2012)]. This type of aggregation approach is not only useful for retail financial products, but can also be used to gather the small and medium size institutions in a banking system, not yet enough concentrated. The aim of this paper is to develop a similar approach of aggregation of individual risks, taking into account not only the frailty effects, but also the contagion effects, in order to analyse the risk of the system.

In our paper, we consider this problem for a transition model in discrete time, at a semiaggregate level with respect to time and individual. Instead of following the dynamics of individual risks, we focus on the dynamics of counts of individuals in the different classes of risk. Therefore, we introduce dynamic models for count processes, with both systematic factors and contagion. The analysis at a semi-aggregate level has several advantages. First, it is less demanding in terms of data confidentiality. Second, it allows for a modelling by means of affine processes, which are tractable for prediction purposes and for studying the stationarity properties. Third, the dynamics at the semi-aggregate level are sufficient for computing portfolio risk measures and pricing credit derivative assets whose payoff is a function of the portfolio loss, such as Collateralized Debt Obligations (CDO).

We consider in Section 2 an homogenous population of individuals with an endogenous dichotomous characteristic following a same homogenous Markov chain. We introduce the counting process defining the number of individuals in state 1 at each date, and show that this is a Markov process with a binomial autoregressive (BinAR) dynamics of order 1. For large population size, alternative limiting processes can be derived, depending whether we apply the Gaussian, or Poisson, approximation of the binomial distribution. This leads to a Gaussian autoregressive, resp. Integer Autoregressive (INAR), approximation. Section 3 explains how correlated risks and contagion can 
be introduced in a BinAR model, and in its two limiting counterparts. We study carefully the stationarity and ergodicity properties of the limiting models. In Section 4 we present the results of some simulation experiments for a logistic model with frailty and contagion, and an INAR model with stochastic intensity. Section 5 concludes. Proofs are gathered in Appendices.

\section{From the homogenous Markov chain to the Gaussian AR(1) and INAR (1) processes}

\subsection{Time-homogenous Markov chain}

Let us denote by $\left(y_{t}, t \in \mathbb{N}\right)$ a time-homogenous Markov chain with two states 0 and 1 . The transition of this chain is characterized by the $2 \times 2$ matrix:

$$
P=\left(\begin{array}{ll}
p_{00} & p_{01} \\
p_{10} & p_{11}
\end{array}\right)
$$

where $p_{i j}=P\left[y_{t}=i \mid y_{t-1}=j\right]$, for $i, j=0,1$. The transition probabilities are such that $p_{i j} \geq 0$, for any $i, j$, and $p_{0 j}+p_{1 j}=1$, for $j=0,1$. The transition matrix admits the eigenvalues 1 and $\rho=p_{00}+p_{11}-1$. Parameter $\rho$ measures the within state stability of the chain. The stationary distribution of the chain $(1-\mu, \mu)^{\prime}$, say, is an eigenvector of transition matrix $P$ associated with the unitary eigenvalue. Parameter $\mu$ is given by:

$$
\mu=\frac{p_{10}}{p_{10}+p_{01}}
$$

and is also equal to the probability to be in state 1 after a change of state.

The transition matrix can be equivalently written either in terms of the transition probabilities, or by means of the two parameters $\rho$ and $\mu$. Indeed, we have:

$$
P=\left(\begin{array}{cc}
1-\mu & 1-\mu \\
\mu & \mu
\end{array}\right)+\rho\left(\begin{array}{cc}
\mu & -(1-\mu) \\
-\mu & 1-\mu
\end{array}\right) .
$$

The transition matrix of the chain at horizon $h$ is:

$$
P^{h}=\left(\begin{array}{cc}
1-\mu & 1-\mu \\
\mu & \mu
\end{array}\right)+\rho^{h}\left(\begin{array}{cc}
\mu & -(1-\mu) \\
-\mu & 1-\mu
\end{array}\right)
$$


This highlights alternative interpretations of parameters $\mu$ and $\rho$, when $|\rho|<1$. Parameter $\mu$ (or equivalently the stationary distribution) is a long run parameter since $\lim _{h \rightarrow \infty} P^{h}=\left(\begin{array}{cc}1-\mu & 1-\mu \\ \mu & \mu\end{array}\right)$, whereas $\rho$ provides the speed of adjustment towards this long run equilibrium. These parameters can be fixed independently: $\mu$ (resp. $\rho$ ) is constrained to be between 0 and 1 (resp. between -1 and 1).

\subsection{The binomial autoregressive (BinAR) process}

Let us now consider an homogenous population of individuals indexed by $i$, for $i=1, \ldots, n$. We assume that the individual state histories $\left(y_{i, t}\right), i=1, \ldots, n$ are independent time-homogeneous Markov chains with the same two-state transition matrix $P$. According to the type of application, states 0 and 1 can have the following interpretations: low risk / high risk (for insured people), default / no default (for corporate loans), investment rating / speculative rating (for sovereign bonds), holder of an insurance contract / not holder (for customers), ill / not ill (for individuals), low liquidity / high liquidity level [for banks, see e.g. Giesecke, Weber (2004), (2006)]. At each date $t$,

we compute the count $N_{t}=\sum_{i=1}^{n} y_{i, t}$ of individuals in state 1 (resp. $n-N_{t}$ in state 0 ), and follow the structure of this homogenous population over time.

To analyse the transition distribution of process $N_{t}$, we note that $N_{t}=N_{1 t}+N_{0 t}$, where $N_{1 t}$ (resp. $N_{0 t}$ ) is the number of individuals in state 1 at date $t-1$ and staying in this state at $t$ (resp. in state 0 at date $t-1$ and changing of state between $t-1$ and $t$ ). Conditional on past individual histories, variables $N_{1 t}$ and $N_{0 t}$ are independent, with conditional binomial distributions:

$$
N_{1 t} \sim \mathcal{B}\left(N_{t-1}, p_{11}\right), \quad N_{0 t} \sim \mathcal{B}\left(n-N_{t-1}, p_{10}\right)
$$

respectively. We deduce the proposition below.

Proposition 1: Under the assumption of independent identically distributed individual Markov chains, the process $\left(N_{t}\right)$ is a Markov process, with values in $\{0,1, \ldots, n\}$ and transition distribution:

$$
\mathcal{B}\left(N_{t-1}, p_{11}\right) * \mathcal{B}\left(n-N_{t-1}, p_{10}\right),
$$

where $*$ denotes the convolution operator. 
In the probabilistic literature, this property is usually written by means of the binomial thinning operator [see Steutel, Van Harn (1979)], defined by

$$
p \circ N=\sum_{i=1}^{N} u_{i},
$$

where $u_{i}$, for $i=1, \ldots, n$, is a sequence of i.i.d. random variables admitting a Bernoulli distribution with parameter $p$, and $N \in \mathbb{N}$. With this notation, we have:

$$
N_{t}=p_{11} \circ N_{t-1}+p_{10} \circ\left(n-N_{t-1}\right),
$$

where the two components of the sum are independent conditional on $N_{t-1}$.

The conditional Laplace transform of count $N_{t}$ is given by:

$$
\begin{aligned}
\psi_{1}(u) & =E_{t-1}\left[\exp \left(-u N_{t}\right)\right] \\
& =\left[1-p_{11}+p_{11} \exp (-u)\right]^{N_{t-1}}\left[1-p_{10}+p_{10} \exp (-u)\right]^{n-N_{t-1}} \\
& =\exp \left\{n \log \left[1-p_{10}+p_{10} \exp (-u)\right]+N_{t-1} \log \left[\frac{1-p_{11}+p_{11} \exp (-u)}{1-p_{10}+p_{10} \exp (-u)}\right]\right\},
\end{aligned}
$$

where $E_{t-1}$ denotes the conditional expectation given the past individual histories. It is defined for $u \geq 0$ and characterizes the transition of the nonnegative process $\left(N_{t}\right)$ [see Feller (1968)]. The conditional Laplace transform is an exponential affine function of lagged count value $N_{t-1}$. Thus, process $\left(N_{t}\right)$ is a compound autoregressive process of order $1[\mathrm{CaR}(1)$, see Darolles, Gourieroux, Jasiak (2006)]. ${ }^{2}$

The transition of process $\left(N_{t}\right)$ at horizon $h$ is $\mathcal{B}\left(N_{t-h}, p_{11}^{(h)}\right) * \mathcal{B}\left(n-N_{t-h}, p_{10}^{(h)}\right)$, where $p_{i j}^{(h)}$ is the $(i, j)$ element of matrix $P^{h}$. From equation (2.3), these elements are given by $p_{11}^{(h)}=\mu+\rho^{h}(1-\mu)$ and $p_{10}^{(h)}=\mu\left(1-\rho^{h}\right)$. In particular, the stationary distribution of $\left(N_{t}\right)$ obtained for $h \rightarrow \infty$, is the binomial distribution $\mathcal{B}(n, \mu)$.

To summarize, count process $\left(N_{t}\right)$ is such that the conditional distributions of both components $N_{1 t}$ and $N_{0 t}$ are binomial, and its unconditional (stationary) distribution is binomial too. This justifies the terminology binomial autoregressive $[\operatorname{Bin} A R(1)]$ process of order 1 . However, the transition of the BinAR(1) process is not binomial itself.

\footnotetext{
${ }^{2} \mathrm{The} \mathrm{CaR}$ processes are called affine processes in the continuous time literature, since the conditional log-Laplace transform is an affine function of the lagged value of the process [see e.g. Duffie, Filipovic, Schachermayer (2003)].
} 


\subsection{The limiting Gaussian AR(1) process}

When the population size is large and the transition probabilities are fixed, the binomial distribution can be approximated by a Gaussian distribution. Equivalently, in terms of processes, the binomial autoregressive process can be approximated by a Gaussian process.

Proposition 2: Let $n \rightarrow \infty$ and transition matrix $P$ be fixed. Assume that $N_{1}$ is such that $X_{n, 1}=$ $\sqrt{n}\left(N_{1} / n-\mu\right)$ converges in distribution to a random variable denoted $\xi_{1}$, and $E\left[X_{n, 1}^{2}\right]=O(1)$. Then, the Markov process $X_{n, t}=\sqrt{n}\left(N_{t} / n-\mu\right)$, for $t \in \mathbb{N}$, converges in distribution to the Gaussian autoregressive process of order 1 , denoted $\left(\xi_{t}\right)$, such that:

$$
\xi_{t}=\rho \xi_{t-1}+\eta \varepsilon_{t}
$$

where $\varepsilon_{t} \sim \operatorname{IIN}(0,1)$ and $\eta^{2}=(1-\mu) p_{10}\left(1-p_{10}\right)+\mu p_{11}\left(1-p_{11}\right)=\mu(1-\mu)\left(1-\rho^{2}\right)$, with initial observation $\xi_{1}$.

Proof: See Appendix 1.

The autoregressive coefficient is equal to the speed of adjustment of the BinAR(1) process. The innovation variance is such that the unconditional distribution of $\left(\xi_{t}\right)$ is $N[0, \mu(1-\mu)]$.

\subsection{The limiting INAR(1) process}

The integer valued autoregressive process (INAR) has been initially introduced in McKenzie (1985) and Al-Osh, Azaid (1987) [see also McKenzie (1988) and Azaid, Al-Osh (1990)]. We derive it below as a limiting case of a binomial autoregressive process, when $p_{01}$ is fixed, but $p_{10}$ tends to zero when the population size $n$ tends to infinity, such that $n p_{10} \sim \lambda$, say, where $\lambda>0$. Under these conditions, we get $p_{11} \sim \rho$ and $n \mu \sim \lambda /(1-\rho)$, and we can use the Poisson approximation of binomial distributions. In particular, the stationary distribution of $N_{t}$ is $\mathcal{P}[\lambda /(1-\rho)]$. It does not explode with $n$; thus, $n-N_{t-1}$ tends to infinity at speed $n$ and the binomial distribution $\mathcal{B}\left(n-N_{t-1}, p_{10}\right)$ is approximately Poisson $\mathcal{P}(\lambda)$, too. We deduce that the limiting process is the INAR(1) process defined below.

Proposition 3: Let $n \rightarrow \infty$ and the transition probabilities be such that $n p_{01} \rightarrow \lambda$, for $\lambda>0$, and $p_{11}$ is fixed. Then, the limiting process is an integer valued autoregressive process of order 
1 [INAR(1)]. Conditionally on the past, $N_{1 t}$ and $N_{0 t}$ are independent with distributions $N_{1 t} \sim$ $\mathcal{B}\left(N_{t-1}, \rho\right)$ and $N_{0 t} \sim \mathcal{P}(\lambda)$, respectively. The stationary distribution of $\left(N_{t}\right)$ is $\mathcal{P}[\lambda /(1-\rho)]$.

The conditional Laplace transform of count $N_{t}$ at horizon 1 is:

$$
\psi_{1}(u)=\exp \left\{-\lambda[1-\exp (-u)]+N_{t-1} \log [1-\rho+\rho \exp (-u)]\right\}
$$

The INAR(1) process is another example of CaR (affine) process of order 1.

\section{Correlated risks versus contagion}

The basic models considered in Sections 2.2-2.4 assume a double homogeneity with respect to both individual and time. In this section, we still assume individual homogeneity, but consider time heterogenous Markov chains, i.e., chains with time dependent transition matrix $P_{t}$, say. We specify $P_{t}$ in order to clearly disentangle correlated risks and contagion.

\subsection{Modelling the correlation vs modelling the contagion}

To illustrate the discussion below, let us interpret the states as not ill/ill. Then, $p_{10}$ is the probability to get the disease for an individual currently in good health, whereas $p_{01}$ is the probability to recover. We focus on a time dependent transition probability $p_{10 t}{ }^{3}$. Thus, we get:

$$
P_{t}=\left(\begin{array}{ll}
p_{00 t} & p_{01} \\
p_{10 t} & p_{11}
\end{array}\right)
$$

i) The dependence between individual risks to get the disease is generally introduced by considering a common stochastic intensity $p_{10 t}$, or equivalently, by assuming that $p_{10 t}=p_{10}\left(F_{t}\right)$, where $F_{t}$ is an unobservable factor ${ }^{4}$ [see e.g. Duffie, Singleton (1998), Schonbucher (2001), Delloy, Fermanian, Sbai (2005), Duffie, Eckner, Horel, Saita (2009) for credit risk applications, and

\footnotetext{
${ }^{3}$ In other applications, such as low liquidity / high liquidity, both $p_{10}$ and $p_{01}$ can depend on time $t$ [see e.g. Giesecke, Weber (2006)].

${ }^{4}$ For a homogenous population, any dependence structure can always be represented by means of such a factor representation [de Finetti (1931), Hewitt, Savage (1955)] with possibly an infinite dimensional factor. In particular, the copula based approach [see e.g. Li (2000), Schonbucher, Schubert (2001)] can be rewritten in this way.
} 
Gschlossl, Czado (2006) for health insurance applications]. This unobservable common factor is called common dynamic frailty in the credit risk literature by analogy with the terminology introduced by Vaupel, Manton, Stallard (1979) for application in demography ${ }^{5}$.

ii) In a homogenous population the contagion effect explains how the number of ill people influences the probability to become sick for individuals currently in good health. This corresponds to a dependence of the type $p_{10 t}=p_{10}\left(N_{t-1}\right)$, where $p_{10}$ is a deterministic function. This is a multivariate extension of Freund model for an homogenous population [Freund (1961)].

In the analysis of social effects, the literature distinguishes in a similar vein between endogenous effects, corresponding to contagion (also called peer effects, neighbourhood effects, herd behaviour, ...), and correlated effects, corresponding to the frailty [see Manski (1993)]. A dynamic binomial model with both risk correlation and contagion is defined by the following assumptions:

Assumption A.1: The individual risk variables $y_{i t}, i=1, \ldots, n$ at date $t$ are independent conditional on the past individual histories $y_{i, t-1}, i=1, \ldots, n$, and on the current and past factor values $\underline{F_{t}}$.

Assumption A.2: The conditional distribution of $y_{i, t}$ depends on individual histories by means of $y_{i, t-1}$ and $N_{t-1}$ only, and on the factor path by means of $F_{t}$ only, respectively. The corresponding

\footnotetext{
${ }^{5}$ The terminology frailty has to be used carefully. In a general framework the transition probability $p_{10, i, t}$ can dependent on both individual $i$ and time $t$, and different unobservable factors can be introduced, that are a pure individual effect $F_{i}$, a pure time effect $F_{t}$, or a joint unobservable effect $F_{i t}$. The standard frailty terminology concerns models with independent individual effects $F_{i}$ [see Greenwood, Yule (1920) for the first introduction of unobserved individual heterogeneity in the literature]. The aim is to account for omitted individual variables and explain the bias due to the fact that less fragile individuals will recover earlier. Joint individual and time effects have been introduced in the microeconometric literature to represent the effort to diminish risk by individuals and capture the moral hazard phenomenon [see Gourieroux, Jasiak (2001) for an application to bonus-malus in motor insurance contracts]. In the framework above, similar to the standard one encountered in the credit risk literature, a stochastic time effect is introduced, that is, all individuals have at a given date the same degree of fragility. This explains the more precise terminology "common dynamic frailty" used in our framework [see also Duffie, Eckner, Horel, Saita (2009), p2096].
} 
transition matrix, conditional on $\underline{F_{t}}$ and $\underline{y_{i, t-1}}, i=1, \ldots, n$, is:

$$
P_{t}=\left(\begin{array}{cc}
1-p_{10}\left(F_{t}, N_{t-1}\right) & p_{01} \\
p_{10}\left(F_{t}, N_{t-1}\right) & p_{11}
\end{array}\right) .
$$

Assumption A.3: The conditional distribution of $F_{t}$ given $\underline{F_{t-1}}, \underline{y_{i, t-1}}, i=1, \ldots, n$, depends on $F_{t-1}$ only, and admits the transition $p d f g\left(f_{t} \mid f_{t-1}\right)$.

Thus, the common factor has an exogenous Markovian dynamics and represents the external shocks influencing the probability to get the disease, such as environmental conditions, whereas contagion is an endogenous phenomenon. The exogenous factor can be multivariate, and we denote by integer $q$ the dimension of vector $F_{t}$.

In the model above, we have implicitly assumed that contagion arises with one lag. This recursive approach avoids the question of simultaneous contagion arising in discrete time models with $p_{10}\left(F_{t}, N_{t}\right)$ [see Manski (1993)] and in continuous time models [see e.g. Jarrow, Yu (2001)], as well as the associated identification problem, often called the reflection problem [Manski (1993)]. We have also assumed that the influences of different sick people are the same. It would be possible to extend the model by assuming that contagion can arise with a limited number of neighbours [see e.g. Giesecke, Weber (2006)], or depend on individual characteristics of sick people.

The conditional distribution of $N_{t}$ given $\underline{F_{t}}, \underline{N_{t-1}}$ becomes:

$$
\mathcal{B}\left(N_{t-1}, p_{11}\right) * \mathcal{B}\left(n-N_{t-1}, p_{10}\left(F_{t}, N_{t-1}\right)\right),
$$

and the joint process $\left(F_{t}, N_{t}\right)$ is Markovian. The conditional distribution of $N_{t}$ given $\underline{N_{t-1}}$ only is derived by integrating out the unobservable factor path. More precisely, this conditional probability is given by:

$$
P\left[N_{t}=n_{t} \mid \underline{N_{t-1}}=\underline{n_{t-1}}, F_{0}=f_{0}\right]=\frac{\int \cdots \int \prod_{\tau=1}^{t} p\left[n_{\tau} \mid n_{\tau-1}, f_{\tau}\right] \prod_{\tau=1}^{t}\left[g\left(f_{\tau} \mid f_{\tau-1}\right) d f_{\tau}\right]}{\int \ldots \int \prod_{\tau=1}^{t-1} p\left[n_{\tau} \mid n_{\tau-1}, f_{\tau}\right) \prod_{\tau=1}^{t-1}\left[g\left(f_{\tau} \mid f_{\tau-1}\right) d f_{\tau}\right]},
$$


where $p\left(n_{t} \mid n_{t-1}, f_{t}\right)$ denotes the elementary probability of the conditional distribution (3.1). The factor integration creates a contemporaneous dependence between individual risks, but also an increase of the memory for count process $\left(N_{t}\right)$, which is no longer Markovian.

Formula (3.2) shows that the effect of lagged counts $N_{t-1}$ on the distribution of current count $N_{t}$ has three origins: i) the dependence of $N_{t}$ on $N_{t-1}$ in the basic binomial autoregressive process; ii) the contagion effect, that is, the dependence of $p_{10 t}$ with respect to $N_{t-1}$; iii) the unobservability of the common dynamic frailty, which introduces the effect of $N_{t-1}$ by means of the filtering distribution of $F_{t}$ given $\underline{N_{t-1}}$. These different effects of $\underline{N_{t-1}}$ corresponding to the transition model, the contagion and the frailty filtering ${ }^{6}$, respectively, can only be identified for special parameterized models.

In finite populations the affine property of process $\left(N_{t}\right)$, and even of joint process $\left(N_{t}, F_{t}\right)$, is not fulfilled in general. We describe in Sections 3.2 and 3.3 below two limiting cases in which the affine property is (partially) recovered. They correspond to the limiting Gaussian AR(1) and INAR(1) processes, respectively. The introduction of correlated risks and contagion in these limiting Gaussian autoregressive and INAR models is deduced by considering a large population and cross aggregating over the population. Thus, the limiting models explain how to pass from a microscopic analysis to a macroscopic one [Fournier, Meleard (2004)].

\subsection{Limiting Gaussian autoregressive model}

i) Let us first consider a model with contagion effect only, that is, $p_{10, t}=p_{10}\left(N_{t-1} / n\right)$, say, where $p_{10}($.$) is a given function. The conditional moments of N_{t} / n$ are:

$$
\begin{aligned}
E_{t-1}\left(N_{t} / n\right) & =p_{11} N_{t-1} / n+\left(1-N_{t-1} / n\right) p_{10}\left(N_{t-1} / n\right) \\
V_{t-1}\left(N_{t} / n\right) & =\frac{1}{n}\left\{p_{11}\left(1-p_{11}\right) N_{t-1} / n+p_{10}\left(N_{t-1} / n\right)\left[1-p_{10}\left(N_{t-1} / n\right)\right]\left(1-N_{t-1} / n\right)\right\} .
\end{aligned}
$$

The conditional variance tends to 0 , when $n$ tends to infinity. This suggests that the variable $N_{t} / n$ converges in quadratic mean to an equilibrium value $\mu$, say, solution of the equation:

$$
\mu=p_{11} \mu+(1-\mu) p_{10}(\mu)
$$

\footnotetext{
${ }^{6}$ See e.g. Collin-Dufresne, Goldstein, Helwege (2003) and Giesecke (2004) for a discussion of the effect of the updating of beliefs.
} 
whenever $p_{10}($.$) is a continuous function. Moreover, if p_{10}($.$) is first-order differentiable, we get:$

$$
\begin{aligned}
p_{10}\left(N_{t-1} / n\right) & \simeq p_{10}(\mu)+\frac{d p_{10}(\mu)}{d \mu}\left(N_{t-1} / n-\mu\right) \\
& =p_{10}(\mu)+\frac{1}{\sqrt{n}} \frac{d p_{10}(\mu)}{d \mu} X_{n, t-1} .
\end{aligned}
$$

This expansion modifies the basic limiting result in Proposition 2 (see Appendix 1). We get the following result:

Corollary 1: Let us consider a pure contagion model with $p_{10 t}=p_{10}\left(N_{t-1} / n\right)$ and define $X_{n, t}=$ $\sqrt{n}\left(N_{t} / n-\mu\right)$, where $\mu$ is the solution of equation (3.3). When $n \rightarrow \infty$, the process $X_{n, t}$ converges in distribution to a process $\xi_{t}^{*}$ such that:

$$
\xi_{t}^{*}=\rho^{*} \xi_{t-1}^{*}+\eta^{*} \varepsilon_{t}^{*}
$$

where $\varepsilon_{t}^{*} \sim \operatorname{IIN}(0,1)$ and:

$$
\begin{aligned}
\eta^{* 2} & =\mu p_{11}\left(1-p_{11}\right)+(1-\mu) p_{10}(\mu)\left[1-p_{10}(\mu)\right] \\
\rho^{*} & =p_{11}-p_{10}(\mu)+(1-\mu) \frac{d p_{10}(\mu)}{d \mu}
\end{aligned}
$$

Let us denote $\Psi_{0}(\mu)=p_{11} \mu+(1-\mu) p_{10}(\mu)$. Thus, the equilibrium value $\mu$ is a fixed point of function $\Psi_{0}$, whereas the limiting autoregressive parameter $\rho^{*}=\frac{d \Psi_{0}(\mu)}{d \mu}$ is the slope of function $\Psi_{0}$ at this point. The Gaussian limiting process $\left(\xi_{t}^{*}\right)$ is stationary if, and only if, the fixed point is locally stable:

$$
\left|\rho^{*}\right|=\left|\frac{d \Psi_{0}(\mu)}{d \mu}\right|<1 .
$$

ii) In the general case with both correlated risks and contagion, we have $p_{10 t}=p_{10}\left(F_{t}, N_{t-1} / n\right)$. By the same arguments as above, $N_{t-1} / n$ tends to a limit $\mu_{t-1}$ when $n$ tends to infinity, where $\mu_{t}$ satisfies the recursive equation:

$$
\mu_{t}=p_{11} \mu_{t-1}+\left(1-\mu_{t-1}\right) p_{10}\left(F_{t}, \mu_{t-1}\right)
$$

which is the analogue of equation (3.3). Due to common factor $F_{t}$, the long run equilibrium at date $t$ is now a dynamic stochastic equilibrium, which depends on the complete factor history. Corollary 1 becomes: 
Corollary 2: Let us consider a model such that $p_{10, t}=p_{10}\left(F_{t}, N_{t-1} / n\right)$ and define $X_{n, t}=$ $\sqrt{n}\left(N_{t} / n-\mu_{t}\right)$, where the stochastic process $\left(\mu_{t}\right)$ of dynamic equilibria satisfies the recursive equation (3.5). When $n \rightarrow \infty$, the process $\left(X_{n, t}\right)$ converges in distribution to a process $\xi_{t}^{*}$ such that:

$$
\xi_{t}^{*}=\rho_{t}^{*} \xi_{t-1}^{*}+\eta_{t}^{*} \varepsilon_{t}^{*}
$$

where $\varepsilon_{t}^{*} \sim \operatorname{IIN}(0,1)$ and:

$$
\begin{aligned}
\eta_{t}^{* 2} & =\mu_{t-1} p_{11}\left(1-p_{11}\right)+\left(1-\mu_{t-1}\right) p_{10}\left(F_{t}, \mu_{t-1}\right)\left[1-p_{10}\left(F_{t}, \mu_{t-1}\right)\right] \\
\rho_{t}^{*} & =p_{11}-p_{10}\left(F_{t}, \mu_{t-1}\right)+\left(1-\mu_{t-1}\right) \frac{\partial p_{10}}{\partial \mu}\left(F_{t}, \mu_{t-1}\right) .
\end{aligned}
$$

We get a 3-dimensional nonlinear state space model, with Markov state vector $\left(\xi_{t}^{*}, \mu_{t}, F_{t}\right)$. It is interesting to understand why the initial 2-dimensional state space $\left(N_{t}, F_{t}\right)$ of the extended dynamic binomial process has been transformed into a 3-dimensional state space in the limiting case. In fact, we have:

$$
N_{t} / n=\mu_{t}+\frac{1}{\sqrt{n}} \xi_{t}^{*}+o(1 / \sqrt{n}) .
$$

Processes $\mu_{t}$ and $\xi_{t}^{*}$ are providing the first two terms in the expansion of $N_{t} / n$ in a neighbourhood of an infinite population size $n \rightarrow \infty$. Specifically, the joint dynamics of $\left(\mu_{t}, F_{t}\right)$ describe the limiting case of infinite population size. These dynamics are studied for some general continuous time models with frailty and contagion by Frey, Backhaus (2003), Giesecke, Weber (2004), (2006). Process $\xi_{t}^{*}$ in Corollary 2 describes the first-order dynamic effect of finite population size. Thus, processes $\mu_{t}$ and $\xi_{t}^{*}$ correspond to the cross-sectionally asymptotic (CSA) and granularity adjustment (GA) components, respectively, in the granularity approach developed in Basel 2 [see e.g. Gordy (2003), (2004), Gagliardini, Gourieroux, Monfort (2012)]. As seen from recursive equation (3.5), $\mu_{t}$ is a deterministic function of the current and lagged factor values. In particular, the sequence $\left(\mu_{t}\right)$ is stochastic, affected at each date by new shocks and does not converge when $t$ tends to infinity. The cross-sectional limiting analysis shows that the sequence of granularity adjustments $\left(\xi_{t}^{*}, t \in \mathbb{N}\right)$ has a simplified dynamics, which is linear Gaussian given the current and lagged values of $\left(F_{t}, \mu_{t}\right)$.

Let us now investigate the strict stationarity and ergodicity properties of the state vector $\left(\xi_{t}^{*}, \mu_{t}, F_{t}\right)$. Let:

$$
F_{t}=a\left(F_{t-1}, \varepsilon_{t}\right),
$$


be the nonlinear autoregressive representation of Markov process $\left(F_{t}\right)$, where $\left(\varepsilon_{t}\right)$ is a white noise process with positive p.d.f. on the support $\mathbb{R}^{m}$.

Assumption A.4: (i) $p_{11}<1$. (ii) The function $p_{10}(f, \mu)$ is differentiable w.r.t. both arguments and $\left\{p_{10}(f, \mu): f \in \mathbb{R}^{q}\right\}=(0,1)$, for any $\mu \in[0,1]$. (iii) The function $a(f, \varepsilon)$ is differentiable w.r.t. both arguments and $\left\{a(f, \varepsilon): \varepsilon \in \mathbb{R}^{m}\right\}=\mathbb{R}^{q}$, for any $f \in \mathbb{R}^{q}$. (iv) The mapping $f \mapsto$ $E\left[\left\|a\left(f, \varepsilon_{t}\right)\right\|\right]$ is continuous on $\mathbb{R}^{q}$. Moreover, there exist real constants $\gamma, c$ and $R$, with $\gamma<1$, such that $E\left[\left\|a\left(f, \varepsilon_{t}\right)\right\|\right] \leq c+\gamma\|f\|$, for $\|f\| \geq R$.

Under Assumption A.4 (ii), the contagion probability can take any value in the interval $(0,1)$ as the exogenous factor varies over its support, for any given equilibrium value $\mu \in[0,1]$. The conditions in Assumptions A.4 (iii) and (iv) are satisfied for instance when $\left(F_{t}\right)$ is a scalar nonlinear autoregressive process with additive error: $F_{t}=\varphi\left(F_{t-1}\right)+\sigma\left(F_{t-1}\right) \varepsilon_{t}$, where the autoregressive function $\varphi$ is such that $|\varphi(f)| \leq c+\gamma|f|$, for $|f|$ large, with $\gamma<1$, the volatility function $\sigma$ is such that $\sigma(f) /|f| \rightarrow 0$ when $|f| \rightarrow \infty$, and $\left(\varepsilon_{t}\right)$ is a strong white noise process with positive p.d.f. on the support $\mathbb{R}$ and $E\left[\left|\varepsilon_{t}\right|\right]<\infty$.

Proposition 4: (i) Under Assumption A.4, the Markov process $X_{t}=\left(\mu_{t}, F_{t}^{\prime}\right)^{\prime}$ defined by equations (3.5) and (3.10) with state space $\mathcal{X}=[0,1] \times \mathbb{R}^{q}$ admits a unique invariant probability measure $\pi$. Moreover, $\left(X_{t}\right)$ is $V$-geometrically ergodic, with function $V$ on $\mathcal{X}$ defined by $V(x)=1+\|f\|$ for $x=\left(\mu, f^{\prime}\right)^{\prime} \in \mathcal{X}$, that is:

$$
\left\|P^{h}(x, \cdot)-\pi\right\|_{V} \leq R V(x) r^{-h}, \quad h \in \mathbb{N}
$$

for some constants $R<\infty$ and $r>1$, and any $x \in \mathcal{X}$, where $P^{h}(x, \cdot)$ denotes the $h$-step transition kernel of Markov process $\left(X_{t}\right)$ with initial value $X_{0}=x$ and $\|\nu\|_{V}:=\sup _{g:|g| \leq V}\left|\int g d \nu\right|$ denotes the $V$-norm of measure $\nu$, with the $\sup$ taken w.r.t. functions $g$ on $\mathcal{X}$ such that $|g(x)| \leq V(x)$ for all $x \in \mathcal{X}$.

(ii) If process $\left(X_{t}\right)$ is initialized with the invariant distribution $X_{0} \sim \pi$, and in addition:

$$
E\left[\log \left|\rho_{t}^{*}\right|\right]<0
$$

where $\rho_{t}^{*}$ is defined in equation (3.8) and the expectation is w.r.t. the invariant probability distribution $\left(\mu_{t-1}, F_{t}^{\prime}\right)^{\prime}$, then process $\left(\xi_{t}^{*}\right)$ defined in (3.6) is strictly stationary. 
Proof: See Appendix 2.

In Proposition 4 (i), the long-term transition kernel $P^{h}(x,$.$) of Markov process \left(X_{t}\right)$ converges to the invariant probability measure $\pi$ at a geometric rate as $h \rightarrow \infty$. This geometric ergodicity property is established by using results in Meyn, Tweedie (2009). In Proposition 4 (ii), the strict stationarity of the granularity adjustment component $\xi_{t}^{*}$ is proved by using a result of Brandt (1986) for linear autoregressive processes with stochastic autoregressive coefficient. The condition (3.11) is the analogue of (3.4) and allows for absolute values of the stochastic autoregressive coefficient larger than 1 at some dates, as long as this does not occur too often.

Different specifications of probability function $p_{10}$ can be introduced. For instance, we can consider a standard probit function with both frailty and lagged count as explanatory variables. This specification arises in the extension of the value of the firm model to contagion effects [see Rosch, Winterfeld (2008) for such an extension in a static framework]. Here we consider a logit specification and focus on three examples with pure contagion, pure frailty effects, and both contagion and frailty effects, respectively.

\section{Example 1: Logistic contagion}

Let us consider a pure contagion model with logistic contagion scheme:

$$
p_{10 t}=p_{10}\left(N_{t-1} / n\right)=\frac{1}{1+\exp \left(-a N_{t-1} / n-b\right)}
$$

This logistic scheme is for instance considered for credit risk analysis in PortfolioView by Mc Kinsey. The long run equilibrium value [see (3.3)] is solution of the equation:

$$
\frac{1-\mu}{\mu} \frac{1}{1+\exp (-a \mu-b)}=1-p_{11}
$$

We prove in Appendix 3 that this solution $\mu=\mu\left(a, b, p_{11}\right)$ exists, is unique and increasing with respect to parameters $a, b, p_{11}$. We also prove that the autoregressive coefficient $\rho^{*}=\rho^{*}\left(a, b, p_{11}\right)$ in Corollary 1 is such that $\left|\rho^{*}\right|<1$ for any values of $a \geq 0, b>0$ and $p_{11} \in(0,1)$. Hence the limit process $\left(\xi_{t}^{*}\right)$ is stationary for any such parameter choice.

In Figure 1 we display the equilibrium value $\mu$ and the autoregressive coefficient $\rho^{*}$ as functions of parameter $a$, for different values of parameters $p_{11}$ and $b$. 
[Insert Figure 1: Equilibrium value and autoregressive coefficient in the logistic model.]

The equilibrium value $\mu$ features an increasing pattern w.r.t. parameter $a$ and approaches the maximum value $1 /\left(2-p_{11}\right)$ when $a$ gets large. The pattern of the autoregressive coefficient $\rho^{*}$ can be non-monotone w.r.t. parameter $a$, and $\rho^{*}$ becomes negative for large $a$. The intuition for negative autoregressive coefficients is the following: For given values of $p_{11}$ and $b$, when parameter $a$ is sufficiently large the contagion probability $\pi_{10}(\mu)$ is such that $\pi_{10}(\mu)>p_{11}$. Then, at equilibrium the contagion probability is larger than the probability to remain sick. Suppose now we move $N_{t-1} / n$ upward from equilibrium such that $N_{t-1} / n>\mu$. Then, the probability of contagion $\pi_{10}\left(N_{t-1} / n\right)$ increases, but the proportion of individuals $1-N_{t-1} / n$ that can be contaged decreases. If the latter effect dominates, on average the proportion of sick individuals will be below the equilibrium, that is, $N_{t} / n<\mu$. Hence, a positive shock on $X_{n, t-1}$ is followed by a negative shock on $X_{n, t}$, which explains the negative autocorrelation coefficient.

\section{Example 2: Pure frailty model}

Let us now consider the limiting model with frailty only. We have $p_{10}\left(F_{t}, \mu_{t-1}\right)=p_{10}\left(F_{t}\right) \equiv$ $F_{t}^{*}$, where the transformed factor process $F_{t}^{*}$ admits values in $(0,1)$. The dynamic equation defining the sequence of equilibria becomes:

$$
\begin{aligned}
\mu_{t} & =p_{11} \mu_{t-1}+\left(1-\mu_{t-1}\right) F_{t}^{*} \\
& =\left(p_{11}-F_{t}^{*}\right) \mu_{t-1}+F_{t}^{*} .
\end{aligned}
$$

In particular, if factor $\left(F_{t}^{*}\right)$ is a strong white noise, the sequence of dynamic equilibria satisfies a bilinear model of order 1 [see Granger and Andersen (1978), Pham, Tran (1981)]. ${ }^{7}$ By recursive substitution, we get:

$$
\mu_{t}=F_{t}^{*}+\sum_{h=1}^{\infty}\left[\prod_{k=0}^{h-1}\left(p_{11}-F_{t-k}^{*}\right)\right] F_{t-h}^{*},
$$

whenever the series in the right hand side exists.

Let us assume that process $\left(F_{t}^{*}\right)$ in $(0,1)$ is strictly stationary and ergodic. Then, the stationarity

\footnotetext{
${ }^{7}$ Process $\left(\mu_{t}\right)$ defined in (3.14) slightly differ from the definition of bilinear process of order 1 adopted in Pham, Tran (1981) since the shock in the stochastic autoregressive coefficient $p_{11}-F_{t}^{*}$ is equal to the innovation $F_{t}^{*}$, and not to its lagged value.
} 
conditions for process $\left(\mu_{t}\right)$ can be derived directly from the results in Brandt (1986) [see also Pham, Tran (1981) and Bougerol, Picard (1992) when $\left(F_{t}^{*}\right)$ is a strong white noise]. Specifically, process $\left(\mu_{t}\right)$ defined in (3.15) is the unique strictly stationary solution of the stochastic recursive equation (3.14) if:

$$
E\left[\log \left|p_{11}-F_{t}^{*}\right|\right]<0
$$

The latter condition is satisfied for any $p_{11} \in[0,1]$. Moreover, when $p_{11}<1$, we have $\mu_{t}<1$ a.s. and equation (3.14) can be solved for $F_{t}^{*}$ to get $F_{t}^{*}=\frac{\mu_{t}-p_{11} \mu_{t-1}}{1-\mu_{t-1}}$. Hence, process $\left(\mu_{t}\right)$ is invertible and the information sets associated with the factor process $\left(F_{t}^{*}\right)$ and the sequence of dynamic equilibria $\left(\mu_{t}\right)$ are the same.

\section{Example 3: Logistic model with contagion and frailty}

We extend the logistic contagion model in Example 1 to include frailty effects as:

$$
p_{10}\left(F_{t}, \mu_{t-1}\right)=\frac{1}{1+\exp \left(-a \mu_{t-1}-b-c F_{t}\right)},
$$

where the parameters are such that $a, c>0$ and $b \in \mathbb{R}$. The scalar exogenous factor $F_{t}$ follows a stationary Gaussian autoregressive process:

$$
F_{t}=\gamma F_{t-1}+\sqrt{1-\gamma^{2}} \varepsilon_{t}
$$

where $\varepsilon_{t} \sim \operatorname{IIN}(0,1)$ and $|\gamma|<1$. The stationary distribution of the exogenous factor is standard Gaussian. Then, Assumption A.4 is satisfied if $p_{11}<1$. From Proposition 4, the Markov process $\left(\mu_{t}, F_{t}\right)$ admits a unique invariant distribution and is geometrically ergodic. We analyze this model by means of Monte-Carlo simulation in Section 4.

\subsection{INAR model with correlated risks and contagion}

When $n p_{10 t}$ is equivalent to $\lambda_{t}=\lambda\left(F_{t}, N_{t-1}\right)$, as $n$ tends to infinity, we get a limiting INAR model with stochastic intensity. The conditional distribution of $N_{t}$ given $N_{t-1}$ and $F_{t}$ is $\mathcal{B}\left(N_{t-1}, \rho\right) *$ $\mathcal{P}\left(\lambda_{t}\right)$. This type of model is especially simple if the stochastic intensity $\lambda_{t}$ is an affine function of both $F_{t}$ and $N_{t-1}$, and moreover $\left(F_{t}\right)$ is itself an affine process. More precisely, let us assume:

Assumption A.5: $\lambda_{t}=c_{0}+c_{1} F_{t}+c_{2} N_{t-1}$, with $c_{0}>0, c_{1} \geq 0$ and $c_{2} \geq 0$. 
Assumption A.6: The scalar factor process is a positive CaR(1) process with conditional Laplace transform:

$$
\psi_{1, t}(u)=E\left[\exp \left(-u F_{t+1}\right) \mid \underline{F_{t}}, \underline{N_{t}}\right]=\exp \left[-\alpha(u) F_{t}-\beta(u)\right]
$$

for some positive functions $\alpha$ and $\beta$.

Since the conditional Laplace transform depends on $F_{t}$ only, the factor features an exogenous dynamics.

Proposition 5: Under Assumptions A.5-A.6, the INAR process with stochastic intensity is such that the bivariate process $\left(N_{t}, F_{t}\right)$ is a CaR(1) process with conditional Laplace transform:

$$
\begin{aligned}
\psi_{1, t}(u, v)= & E\left[\exp \left(-u N_{t+1}-v F_{t+1}\right) \mid \underline{N_{t}}, \underline{F_{t}}\right] \\
= & \exp \left\{-c_{0}[1-\exp (-u)]-\beta\left(v+c_{1}[1-\exp (-u)]\right)\right. \\
& \left.-N_{t}\left(c_{2}[1-\exp (-u)]-\log [1-\rho+\rho \exp (-u)]\right)-F_{t} \alpha\left(v+c_{1}[1-\exp (-u)]\right)\right\} .
\end{aligned}
$$

Proof: See Appendix 4.

The main advantage of the specification above is the simple characterization of the case with correlated risks only (resp. contagion only), which corresponds to the restriction $c_{2}=0$ (resp. $\left.c_{1}=0\right)$. These hypotheses can be easily tested in practice once a parametric specification is chosen for the factor dynamics (see below). Another advantage of a CaR process is to provide easily nonlinear predictions at any horizon. More precisely, the conditional Laplace transform at horizon $h$ is:

$$
\begin{aligned}
\psi_{h, t}(u, v) & =E\left[\exp \left(-u N_{t+h}-v F_{t+h}\right) \mid \underline{N_{t}}, \underline{F_{t}}\right] \\
& =\exp \left[-a_{1, h}(u, v) N_{t}-a_{2, h}(u, v) F_{t}-b_{h}(u, v)\right]
\end{aligned}
$$

where $a_{1, h}, a_{2, h}$ and $c_{h}$ are computed by recursion

$$
\begin{aligned}
a_{1, h}(u, v) & =c_{2}\left[1-\exp \left(-a_{1, h-1}(u, v)\right)\right]-\log \left[1-\rho+\rho \exp \left(-a_{1, h-1}(u, v)\right)\right] \\
a_{2, h}(u, v) & =\alpha\left\{a_{2, h-1}(u, v)+c_{1}\left[1-\exp \left(-a_{1, h-1}(u, v)\right)\right]\right\} \\
b_{h}(u, v) & =b_{h-1}(u, v)+c_{0}\left[1-\exp \left(-a_{1, h-1}(u, v)\right)\right]+\beta\left\{a_{2, h-1}(u, v)+c_{1}\left[1-\exp \left(-a_{1, h-1}(u, v)\right)\right]\right\}
\end{aligned}
$$


where:

$$
\begin{aligned}
& a_{1,1}(u, v)=c_{2}[1-\exp (-u)]-\log [1-\rho+\rho \exp (-u)], \quad a_{2,1}(u, v)=\alpha\left(v+c_{1}[1-\exp (-u)]\right), \\
& b_{1}(u, v)=c_{0}[1-\exp (-u)]+\beta\left(v+c_{1}[1-\exp (-u)]\right) .
\end{aligned}
$$

Moreover, by considering the behaviour of functions $a_{1,1}(u, v)$ and $a_{2,1}(u, v)$ in a neighbourhood of $u=v=0$, the stationarity conditions of the joint process $\left(N_{t}, F_{t}\right)$ are directly deduced [see Darolles, Gourieroux, Jasiak (2006), Proposition 6.2]. More precisely, the joint process $\left(N_{t}, F_{t}\right)$ is strictly stationary if, and only if, the modulus of the eigenvalues of the matrix:

$$
\left(\begin{array}{ll}
\frac{\partial a_{1,1}}{\partial u}(0,0) & \frac{\partial a_{1,1}}{\partial v}(0,0) \\
\frac{\partial a_{2,1}}{\partial u}(0,0) & \frac{\partial a_{2,1}}{\partial v}(0,0)
\end{array}\right)=\left(\begin{array}{cc}
c_{2}+\rho & 0 \\
\alpha^{\prime}(0) c_{1} & \alpha^{\prime}(0)
\end{array}\right)
$$

are strictly smaller than 1 . We get the stationarity conditions in the next result.

Proposition 6: Under Assumptions A.5-A.6, the Markov process $\left(N_{t}, F_{t}\right)$ is strictly stationary if, and only if:

$$
c_{2}+\rho<1, \quad \alpha^{\prime}(0)<1
$$

The condition $\alpha^{\prime}(0)<1$ is the stationarity condition for the $\mathrm{CaR}(1)$ process $\left(F_{t}\right)$ in Assumption A.6, while the condition $c_{2}+\rho<1$ involves the autoregressive parameter $\rho$ of the INAR process and the parameter $c_{2}$ that describes the contagion effect in the stochastic intensity.

We can derive further results concerning the distribution of process $\left(N_{t}, F_{t}\right)$ if we assume that the factor is an autoregressive Gamma process (ARG) of order 1 [Gourieroux, Jasiak (2006)], that is, the $\mathrm{CaR}(1)$ process which is a time discretized Cox, Ingersoll, Ross process [Cox, Ingersoll, Ross (1985)]. The corresponding conditional Laplace transform is given by:

$$
E\left[\exp \left(-u F_{t+1}\right) \mid F_{t}\right]=\frac{1}{(1+\eta u)^{\delta}} \exp \left(-\frac{\gamma u}{1+\eta u} F_{t}\right)
$$

that is,

$$
\alpha(u)=\frac{\gamma u}{1+\eta u}, \quad \beta(u)=\delta \log (1+\eta u),
$$

where $\gamma \geq 0$ and $\delta, \eta>0$. Parameter $\gamma$ is the first-order autocorrelation of process $\left(F_{t}\right)$. The unconditional distribution of $F_{t}$ is a gamma distribution with parameters $(\delta, \eta /(1-\gamma))$. The 
component $N_{0 t}$ follows a Poisson distribution with gamma heterogeneity, that is, a negative binomial distribution. Thus, by introducing an ARG factor, we transform the initial process based on Poisson distributions in a process based on negative binomial distributions [see e.g. Bockenholt (1999)] and solve the standard overdispersion problem in a dynamic framework [Greenwood, Yule (1920)]. The sensitivity parameter $c_{1}$ in the intensity and the scale parameter $\eta$ of the factor $F_{t}$ cannot be identified separately. For instance, we can set parameter $\eta$ such that $E\left[F_{t}\right]=1$. Since $E[F]=\delta \eta /(1-\gamma)$ [see Gourieroux, Jasiak (2006)], we can assume $\eta=(1-\gamma) / \delta$.

From Proposition 6, process $\left(N_{t}, F_{t}\right)$ is strictly stationary if, and only if, $c_{2}+\rho<1$ and $\gamma<1$. The first- and second-order moments of the stationary distribution are given in the next proposition, proved in Appendix 4.

Proposition 7: Let Assumptions A.5-A.6 hold, and let the factor $F_{t}$ follow the ARG(1) process as in (3.22)-(3.23). Then, when $\eta=(1-\gamma) / \delta$, the unconditional means, variances and covariance of process $\left(N_{t}, F_{t}\right)$ are given by:

$$
\begin{gathered}
E\left[N_{t}\right]=\frac{c_{0}+c_{1}}{1-\left(c_{2}+\rho\right)}, \quad E\left[F_{t}\right]=1, \\
V\left[N_{t}\right]=\frac{c_{0}+c_{1}}{1-\left(c_{2}+\rho\right)}\left[\frac{1-\rho^{2}}{1-\left(c_{2}+\rho\right)^{2}}\right]+\frac{c_{1}^{2}}{\delta} \frac{1}{1-\left(c_{2}+\rho\right)^{2}}\left[\frac{1+\gamma\left(c_{2}+\rho\right)}{1-\gamma\left(c_{2}+\rho\right)}\right], \quad V\left[F_{t}\right]=\frac{1}{\delta},
\end{gathered}
$$

and:

$$
\operatorname{Cov}\left(F_{t}, N_{t}\right)=\frac{c_{1}}{\delta} \frac{1}{1-\gamma\left(c_{2}+\rho\right)}
$$

The stationary distribution of the count variable $N_{t}$ features overdispersion, that is, $V\left[N_{t}\right]>E\left[N_{t}\right]$, when either $c_{1}>0$, or $c_{2}>0$ (or both). In the first case, overdispersion is due to the frailty effect, while in the second case it is due to the contagion effect. The processes $\left(N_{t}\right)$ and $\left(F_{t}\right)$ feature a positive contemporaneous unconditional correlation when $c_{1}>0$.

Proposition 8 provides the autocorrelogram of the count process $\left(N_{t}\right)$.

Proposition 8: Let Assumptions A.5-A.6 hold, and let the factor $F_{t}$ follow the ARG(1) process as in (3.22)-(3.23) with $\eta=(1-\gamma) / \delta$. Then, the autocorrelogram of process $\left(N_{t}\right)$ is such that:

$$
\operatorname{Corr}\left(N_{t+h}, N_{t}\right)=(1-\omega)\left(c_{2}+\rho\right)^{h}+\omega \gamma^{h}, \quad h \geq 0
$$


where $\omega=\frac{c_{1} \gamma}{\gamma-\left(c_{2}+\rho\right)} \frac{\operatorname{Cov}\left(N_{t}, F_{t}\right)}{V\left(N_{t}\right)}$, if $\gamma \neq c_{2}+\rho$, and:

$$
\operatorname{Corr}\left(N_{t+h}, N_{t}\right)=(1+\tilde{\omega} h) \gamma^{h}, \quad h \geq 0,
$$

where $\tilde{\omega}=c_{1} \frac{\operatorname{Cov}\left(N_{t}, F_{t}\right)}{V\left(N_{t}\right)}$, if $\gamma=c_{2}+\rho$.

Proof: See Appendix 4.

The autocorrelogram of process $\left(N_{t}\right)$ decays geometrically w.r.t. the lag. Indeed, as a consequence of the $\mathrm{CaR}(1)$ property of the joint process $\left(N_{t}, F_{t}\right)$ in Proposition 5 , the conditional mean of $\left(N_{t}, F_{t}\right)^{\prime}$ given the past $\left(\underline{N_{t-1}}, \underline{F_{t-1}}\right)$ is a linear function of the lagged value $\left(N_{t-1}, F_{t-1}\right)^{\prime}$, as in a bivariate VAR process (see Lemma A.8 in Appendix 4). The associated matrix of the autoregressive coefficients is the transposed of the matrix in equation (3.20), whose eigenvalues are $c_{2}+\rho$ and $\gamma$. Hence, when the eigenvalues $c_{2}+\rho$ and $\gamma$ are distinct, the autocorrelogram of $\left(N_{t}\right)$ is a linear combination of the autocorrelogram $\left(c_{2}+\rho\right)^{h}, h \in \mathbb{N}$, of the INAR process with pure contagion, and autocorrelogram $\gamma^{h}, h \in \mathbb{N}$, of the ARG process $\left(F_{t}\right)$. When the two eigenvalues $c_{2}+\rho$ and $\gamma$ are equal, the autocorrelogram of $\left(N_{t}\right)$ involves also a multiplicative term that is linear in the lag.

\section{Example 4: Pure correlated risks}

When only correlated risks $\lambda_{t}=c_{0}+c_{1} F_{t}$ are introduced, we get a recursive system in which the factor dynamics is fixed exogenously, then driving the dynamics of the count process. This allows for computing nonlinear predictions in two steps, first by considering the conditional distribution of $N_{t+h}$ given $\underline{F_{t+h}}, \underline{N_{t}}$, then by reintegrating out the future factor path given $\underline{F_{t}}, \underline{N_{t}}$. We have:

$$
\begin{aligned}
& E\left[\exp \left(-u N_{t+h}\right) \underline{F_{t+h}}, \underline{N_{t}}\right] \\
= & \exp \left\{-\left(\lambda_{t+h}+\rho \lambda_{t+h-1}+\ldots+\rho^{h-1} \lambda_{t+1}\right)[1-\exp (-u)]+N_{t} \log \left[1-\rho^{h}+\rho^{h} \exp (-u)\right]\right\} \\
= & \exp \left\{-\left[c_{0} \frac{1-\rho^{h}}{1-\rho}+c_{1}\left(F_{t+h}+\rho F_{t+h-1}+\ldots+\rho^{h-1} F_{t+1}\right)\right][1-\exp (-u)]\right. \\
& \left.+N_{t} \log \left[1-\rho^{h}+\rho^{h} \exp (-u)\right]\right\} .
\end{aligned}
$$

We deduce that:

$$
\begin{aligned}
E\left[\exp \left(-u N_{t+h}\right) \mid \underline{F_{t}}, \underline{N_{t}}\right]= & \exp \left\{-c_{0} \frac{1-\rho^{h}}{1-\rho}[1-\exp (-u)]+N_{t} \log \left[1-\rho^{h}+\rho^{h} \exp (-u)\right]\right\} \\
& E\left[\exp \left\{-c_{1}\left(F_{t+h}+\rho F_{t+h-1}+\ldots+\rho^{h-1} F_{t+1}\right)[1-\exp (-u)]\right\} \mid F_{t}\right] .
\end{aligned}
$$


To conclude this computation, we have to explain how to compute recursively the nonlinear prediction of the smoothed future path $F_{t+h}+\rho F_{t+h-1}+\ldots+\rho^{h-1} F_{t+1}$. It is easily checked that:

$$
E\left\{\exp \left[-v\left(F_{t+h}+\rho F_{t+h-1}+\ldots+\rho^{h-1} F_{t+1}\right)\right] \mid F_{t}\right\}=\exp \left[-a_{h}(v) F_{t}-b_{h}(v)\right]
$$

where $a_{h}$ and $c_{h}$ satisfy the recursive equations:

$$
a_{h}(v)=\alpha\left[a_{h-1}(v)+v \rho^{h-1}\right], \quad b_{h}(v)=b_{h-1}(v)+\beta\left[a_{h-1}(v)+v \rho^{h-1}\right],
$$

with initial conditions:

$$
a_{1}(v)=\alpha(v), \quad b_{1}(v)=\beta(v)
$$

Thus, we get:

$$
\begin{aligned}
E\left[\exp \left(-u N_{t+h}\right) \mid \underline{F_{t}}, \underline{N_{t}}\right]= & \exp \left\{-c_{0} \frac{1-\rho^{h}}{1-\rho}[1-\exp (-u)]+N_{t} \log \left[1-\rho^{h}+\rho^{h} \exp (-u)\right]\right. \\
& \left.-F_{t} a_{h}\left(c_{1}[1-\exp (-u)]\right)-b_{h}\left(c_{1}[1-\exp (-u)]\right)\right\}
\end{aligned}
$$

\section{Simulation experiments}

In this Section we report the results of simulation experiments in two dynamic models with both contagion and correlated risks. The first model is a logistic specification admitting a limit Gaussian approximation. The second model is the INAR process with stochastic intensity.

\subsection{Logistic model with contagion and correlated risks}

The contagion probability admits a logistic specification as in equation (3.17), where the parameters are $a=5, b=-\log (9)$ and $c=2$. The probability of staying in state 1 is $p_{11}=0.5$. The factor $\left(F_{t}\right)$ follows a Gaussian autoregressive process as in equation (3.18), where the autoregressive coefficient is $\gamma=0.5$. The number of individuals is $n=100$.

In Figure 2 we display simulated paths for the factor $F_{t}$, the proportion $N_{t} / n$ of individuals in state 1 and the stochastic equilibrium $\mu_{t}$.

[Insert Figure 2: Simulated paths of factor, count and stochastic equilibrium in the logistic model with contagion and correlated risks.] 
The path of $N_{t} / n$ is close to that of the stochastic equilibrium $\mu_{t}$, that is, the CSA approximation, although the path of $\mu_{t}$ is smoother. The dynamics of the equilibrium $\mu_{t}$ features regimes that are driven by factor $F_{t}$. When the values of factor $F_{t}$ are close to zero, the equilibrium $\mu_{t}$ is close to 0.6. When the factor $F_{t}$ features negative shocks, the equilibrium $\mu_{t}$ decreases sharply. Positive shocks on $F_{t}$ are associated with rather small increases in $\mu_{t}$. Hence, the reaction of the dynamic equilibrium $\mu_{t}$ to positive and negative shocks in $F_{t}$ is asymmetric.

Figure 3 displays the scatter plot of a simulated path of the joint equilibrium and factor process $\left(\mu_{t}, F_{t}\right)$. From Example 3 in Section 3.2, this process is strictly stationary, if initialized with the unique invariant distribution. The shaded area in Figure 3 is the support of the stationary distribution, that is derived in Proposition A.1 in Appendix A.2.1.

[Insert Figure 3: Scatter plot and support of the stationary distribution of the joint equilibrium and factor process for the logistic model with contagion and correlated risks.]

The support of the stationary distribution is a strict subset of $[0,1] \times \mathbb{R}$. In particular, the support contains neither equilibrium values $\mu_{t}>p_{11}$ associated with small negative realizations of the factor $F_{t}$, nor equilibrium values $\mu_{t}<p_{11}$ associated with large positive realizations of the factor $F_{t}$. Many points in the scatter plot cumulate in a state space region with factor value around zero and equilibrium value close to 0.6, as already observed in Figure 2.

Figure 4 displays simulated paths for the standardized deviation from the equilibrium $X_{n, t}=$ $\sqrt{n}\left(N_{t} / n-\mu_{t}\right)$, the autoregressive coefficient $\rho_{t}^{*}$ and the volatility $\eta_{t}^{*}$ of the Gaussian approximation. The corresponding simulated path of factor values is the same as in Figure 2.

[Insert Figure 4: Simulated paths of deviation from equilibrium, autoregressive coefficient and volatility of the Gaussian approximation for the logistic model with contagion and correlated risks.]

The path of $X_{n, t}$ features regimes in persistency, with both periods of positive autocorrelation and periods of negative autocorrelation. This is reflected in the dynamics of the autoregressive coefficient $\rho_{t}^{*}$ of the Gaussian approximation, that admits both values slightly above 1 and negative values. The path of volatility $\eta_{t}^{*}$ is more stable than that of the autoregressive coefficient $\rho_{t}^{*}$, and features some sharp downward movements associated with the negative shocks on the factor $F_{t}$. For the selected model parameter values, we compute the expectation $E\left[\log \left|\rho_{t}^{*}\right|\right]$ by Monte- 
Carlo simulation and get $E\left[\log \left|\rho_{t}^{*}\right|\right]=-1.0893$. Hence, condition (3.11) is satisfied and from Proposition 4 process $\left(\rho_{t}^{*}\right)$ is strictly stationary.

In order to assess the accuracy of the approximation (3.9), let us consider the standardized residuals:

$$
\tilde{\varepsilon}_{t}^{*}=\frac{X_{n, t}-\rho_{t}^{*} X_{n, t-1}}{\eta_{t}^{*}}, \quad t \text { varying, }
$$

that are the residuals for the autoregressive process in (3.6) computed from process $X_{n, t}$. If the approximation (3.9) is accurate, process $\tilde{\varepsilon}_{t}^{*}$ is close to a standard Gaussian white noise. We display some summary statistics for the unconditional distribution as well as autocorrelations for process $\tilde{\varepsilon}_{t}^{*}$ in Table 1 . They are computed by Monte-Carlo on a long simulated path of the process.

[Insert Table 1: Summary statistics and autocorrelogram of process $\tilde{\varepsilon}_{t}^{*}$ ]

We consider different population sizes, that are $n=25, n=100$ and $n=1000$. From Table 1 it is seen that process $\tilde{\varepsilon}_{t}^{*}$ gets closer to a standard Gaussian white noise when $n$ increases, which confirms that the accuracy of approximation (3.9) improves with the population size.

\subsection{INAR model with stochastic intensity}

Let us now consider an INAR model with stochastic intensity as in Assumptions A.5-A.6. The exogenous factor $F_{t}$ follows an ARG process with autocorrelation parameter $\gamma=0.5$ and shape parameter $\delta=2$. The scale parameter $\eta$ is set equal to $\eta=(1-\gamma) / \delta=0.25$ to get $E\left[F_{t}\right]=1$ [see Section 3.3]. The autoregressive parameter $\rho$ of the INAR process is $\rho=0.2$. Moreover, we consider four parameter sets for the intensity specification:

A) $c_{0}=2.4, c_{1}=0, c_{2}=0$

B) $c_{0}=1.4, c_{1}=1, c_{2}=0$,

C) $c_{0}=1.2, c_{1}=0, c_{2}=0.4$,

D) $c_{0}=0.2, c_{1}=1, c_{2}=0.4$.

Parameters $c_{1}$ and $c_{2}$ are selected such that models $\mathrm{A}, \mathrm{B}, \mathrm{C}$, and $\mathrm{D}$ correspond to specifications with constant intensity, pure frailty effect, pure contagion effect, and both frailty and contagion 
effects, respectively. The parameter $c_{0}$ is selected to have the same unconditional mean for count $N_{t}$ across all specifications, which is equal to $E\left[N_{t}\right]=\frac{c_{0}+c_{1}}{1-\left(\rho+c_{2}\right)}=3$.

\section{i) Simulated paths}

Let us first compare simulated paths of the process $\left(N_{t}\right)$ for the different parameter sets AD. For this purpose, it is useful to rewrite the model in the nonlinear autoregressive stochastic representation:

$N_{t}=\inf \left\{m \in \mathbb{N}, m \leq N_{t-1}: \sum_{j=0}^{m} \pi_{j, t}^{(1)} \geq U_{1, t}\right\}+\inf \left\{m \in \mathbb{N}: \sum_{j=0}^{m} \pi_{j, t}^{(2)} \geq U_{2, t}\right\} \equiv a\left(N_{t-1}, F_{t}, U_{t}\right)$,

where $\pi_{j, t}^{(1)}=\frac{N_{t-1} !}{j !\left(N_{t-1}-j\right) !} \rho^{j}(1-\rho)^{N_{t-1}-j}$ and $\pi_{j, t}^{(2)}=e^{-\lambda_{t}} \frac{\lambda_{t}^{j}}{j !}$, with $\lambda_{t}=c_{0}+c_{1} F_{t}+c_{2} N_{t-1}$, are the probability weights for the conditional binomial and Poisson distributions $\mathcal{B}\left(N_{t-1}, \rho\right)$ and $\mathcal{P}\left(\lambda_{t}\right)$ given $N_{t-1}$ and $F_{t}$, respectively, and variables $U_{t}=\left(U_{1, t}, U_{2, t}\right)$ are i.i.d. such that $U_{1, t}$ and $U_{2, t}$ are independent with uniform distribution $\mathcal{U}[0,1]$. The distribution of the factor $\left(F_{t}\right)$ and shocks $\left(U_{t}\right)$ is independent of intensity parameters $c_{0}, c_{1}$ and $c_{2}$. This allows us to compare the simulated paths of process $\left(N_{t}\right)$ obtained from a same path of $\left(F_{t}, U_{t}\right)$ and different intensity parameters $c_{0}, c_{1}, c_{2}$ as in sets A-D above.

The simulated path of $\left(N_{t}\right)$ for parameter sets A-B and C-D are displayed in Figures 5 and 6, respectively.

[Insert Figure 5: Simulated paths of the INAR process with stochastic intensity (parameter sets A and B)]

[Insert Figure 6: Simulated paths of the INAR process with stochastic intensity (parameter sets C and D)]

Simulated paths can be used to discuss the identification power of several summary statistics.

\section{ii) Conditional expectation}

In Figure 7 we display the conditional expectation of $N_{t}$ given $N_{t-1}=n_{t-1}$ as a function of lagged value $n_{t-1}$ for parameter sets A-D. 
[Insert Figure 7: Conditional expectation of $N_{t}$ given $N_{t-1}$ in the INAR process with stochastic intensity]

The conditional expectation is linear $E\left[N_{t} \mid N_{t-1}\right]=c_{0}+\left(\rho+c_{2}\right) N_{t-1}=E\left[N_{t}\right]+\left(\rho+c_{2}\right)\left(N_{t-1}-\right.$ $E\left[N_{t}\right]$ ) for models with constant intensity, or pure contagion effects (parameter sets $\mathrm{A}$ and $\mathrm{C}$ ). For models including frailty effects (parameter sets B and D), we compute the conditional expectation $E\left[N_{t} \mid N_{t-1}\right]$ by Monte-Carlo on a long simulated path of process $\left(N_{t}\right)$. From Figure 7 it is seen that the conditional expectation is close to linear also for parameter sets B and D. Moreover, models A, B, C and D are ranked in order of increasing (linear, first-order) persistency. However, model C with pure contagion cannot be distinguished from a model with constant intensity and autoregressive INAR parameter $\tilde{\rho}=\rho+c_{2}$ based on the conditional expectation at lag one. Figure 7 suggests that this is hardly possible also for models B and D including frailty effects.

\section{iii) Autocorrelogram}

From Proposition 8 the autocorrelogram of $\left(N_{t}\right)$ is a mixture of two power functions of $\gamma$ and $c_{2}+\rho$, respectively. Thus, we expect to better identify contagion and frailty effects from the ACF, at least when parameters $\gamma$ and $c_{2}+\rho$ are both non-zero and sufficiently different. More precisely, the $\log$ ACF is nonlinear if, and only if, $c_{1}>0$ and $\gamma>0$, that is, if there is a dynamic frailty effect. The nonlinearity is weak when the autocorrelation coefficient of the frailty $\gamma$ is close to $c_{2}+\rho$. There is no nonlinearity at all when the frailty is static.

In Figure 8 we display the autocorrelogram $\left\{\operatorname{Corr}\left(N_{t+h}, N_{t}\right), h \in \mathbb{N}\right\}$ of process $\left(N_{t}\right)$ for parameter sets A-D.

[Insert Figure 8: Autocorrelogram of the INAR process $\left(N_{t}\right)$ with stochastic intensity]

For experiments $\mathrm{A}$ and $\mathrm{C}$ with no frailty effect, the $\log \mathrm{ACF}$ is linear. At the opposite, some curvature of the $\log \mathrm{ACF}$ is observed for experiment B with pure frailty, where $c_{2}+\rho=0.2$ and $\gamma=0.5$. The $\log$ ACF is almost linear for experiment $\mathrm{D}$ with both frailty and contagion, where $c_{2}+\rho=0.6$ and $\gamma=0.5$. 


\section{iv) Conditional overdispersion}

Let us now investigate whether the analysis of higher-order moments of the conditional distribution of $N_{t+1}$ given $N_{t}$ can be useful for identifying contagion vs frailty effects. The overdispersion coefficient of the conditional distribution of $N_{t+1}$ given $N_{t}$ is defined as the ratio between the conditional variance $V\left[N_{t+1} \mid N_{t}\right]$ and the conditional mean $E\left[N_{t+1} \mid N_{t}\right]$. In Appendix 4 we show that:

$$
V\left[N_{t+1} \mid N_{t}\right]=E\left[N_{t+1} \mid N_{t}\right]-\rho^{2} N_{t}+c_{1}^{2}\left(\gamma^{2} V\left[F_{t} \mid N_{t}\right]+2 \frac{\gamma(1-\gamma)}{\delta} E\left[F_{t} \mid N_{t}\right]+\frac{(1-\gamma)^{2}}{\delta}\right)
$$

where:

$$
E\left[N_{t+1} \mid N_{t}\right]=E\left[N_{t}\right]+\left(c_{2}+\rho\right)\left(N_{t}-E\left[N_{t}\right]\right)+c_{1} \gamma\left(E\left[F_{t} \mid N_{t}\right]-1\right)
$$

Hence, a model without frailty effect $\left(c_{1}=0\right)$ features conditional underdispersion. Intuitively, this is because the conditional distribution is expected to feature less overdispersion than the marginal distribution, and the latter is Poisson, i.e., with zero overdispersion. The contribution of frailty to conditional dispersion is positive. It involves the conditional mean $E\left[F_{t} \mid N_{t}\right]$ and variance $V\left[F_{t} \mid N_{t}\right]$ of the unobservable factor $F$ given the observable count $N_{t}$, as well as the unconditional variance $V\left[F_{t}\right]=1 / \delta$ of the factor and its autocorrelation parameter $\gamma$. When the sensitivity parameter $c_{1}$ is large enough, the positive frailty effect can dominate and yield conditional overdispersion (at least for some lag $N_{t}$ ).

In Figure 9 we display the conditional overdispersion of $N_{t+1}$ given $N_{t}$ as a function of lagged value $N_{t}$ for parameter sets A-D.

[Insert Figure 9: Conditional overdispersion of $N_{t+1}$ given $N_{t}$ in the INAR process with stochastic intensity]

The introduction of a frailty effect has a positive level effect on the conditional overdispersion function. As expected, specifications $\mathrm{A}$ and $\mathrm{C}$ feature conditional underdispersion, while specifications $\mathrm{B}$ and $\mathrm{D}$ feature conditional overdispersion for all displayed conditioning values $N_{t}$. The introduction of a contagion effect has a slope effect on the conditional overdispersion function. For instance, comparing the patterns for specifications $\mathrm{C}$ and $\mathrm{D}$, the slope of the conditional overdispersion function is more negative for specification D. 


\section{Conclusions}

In this paper we analyze frailty correlated risks and contagion effects in large homogeneous populations. We consider a microscopic dynamic model in which individual risks can take two states (high and low), and the individual transition probabilities between states are time varying and stochastic. The frailty effect is modeled by means of a common unobservable factor $F_{t}$ that impacts the individual transition probabilities. The contagion effect is modeled through the dependence of the individual transition probabilities on the lagged count $N_{t-1}$ of individuals in the high risk state. We derive macroscopic models for the count process $\left(N_{t}\right)$ as the limit of the microscopic model when the population size $n$ tends to infinity. Different macroscopic dynamics are obtained according to whether the transition probabilities are assumed fixed w.r.t. $n$ (Gaussian approximation), or the transition probability to the high risk state converges to zero when $n$ increases (Poisson approximation). In the first setting, we derive an approximation for the dynamics of the proportion $N_{t} / n$ in terms of a dynamic stochastic equilibrium driven by factor $F_{t}$ plus a granularity adjustment at order $1 / \sqrt{n}$ involving a conditionally Gaussian autoregressive process. In the second setting, we carefully study the properties of the INAR process for count $N_{t}$ with stochastic intensity driven by factor $F_{t}$ and lagged count $N_{t-1}$.

An interesting question is to which extent it is possible to identify frailty and contagion effects from the macroscopic dynamics only. The analysis of the INAR model with stochastic intensity suggests that it can be rather difficult to disentangle these two effects when relying solely on summaries of the process that capture linear dynamics, such as the conditional mean function or the autocorrelogram of process $\left(N_{t}\right)$. Instead, nonlinear features of the conditional distribution of $N_{t}$ given $N_{t-1}$, such as the conditional overdispersion function, can be very useful to disentangle frailty and contagion effects. 


\section{References}

[1] Ait-Sahalia, Y., Cacho-Diaz, J., and R., Laeven (2010): "Modeling Financial Contagion Using Mutually Exciting Jump Processes”, DP Princeton University.

[2] Al-Osh, M., and A., Azaid (1987): "First-Order Integer Valued Autoregressive [INAR(1)] Processes", Journal of Time Series Analysis, 8, 261-275.

[3] Azaid, A., and M., Al-Osh (1990): "An Integer Valued Path Order Autoregressive Structure [INAR(p)] Process", Journal of Applied Probability, 27, 314-324.

[4] Azizpour, S., and K., Giesecke (2008)a: "Premia for Correlated Default Risk ”, DP Stanford University.

[5] Azizpour, S., and K., Giesecke (2008)b: "Self-exciting Corporate Defaults, Contagion vs Frailty“, DP Stanford University.

[6] Bailey, N. (1953): “The Total Size of a General Stochastic Epidemic”, Biometrika, 40, 177185.

[7] Bailey, N. (1957): The Mathematical Theory of Epidemics, Charles Griffin, London.

[8] Basel Committee on Banking Supervision (2001): “The New Basel Capital Accord”, Consultative Document of the Bank for International Settlements, April 2001, Part 2: Pillar 1.

[9] Basel Committee on Banking Supervision (2003): “The New Basel Capital Accord”, Consultative Document of the Bank for International Settlements, April 2003, Part 3: The Second Pillar.

[10] Billingsley, P. (1968): Convergence of Probability Measures, Wiley Series in Probability and Mathematical Statistics, John Wiley and Sohns, New York.

[11] Bockenholt, U. (1999): "An INAR(1) Negative Multinomial Regression Model For Longitudinal Count Data", Psychometrika, 64, 53-67.

[12] Bougerol, P., and N., Picard (1992): "Strict Stationarity of Generalized Autoregressive Processes“, Annals of Probability, 20, 1714-1730. 
[13] Brandt, A. (1986): "The Stochastic Equation $Y_{n+1}=A_{n} Y_{n}+B_{n}$ with Stationary Coefficients“, Advances in Applied Probability, 18, 211-220.

[14] Collin-Dufresne, P., Goldstein, R., and J., Helwege (2003): 'Is Credit Event Risk Priced? Modeling Contagion via the Updating of Belief", DP Haas School, University of California, Berkeley.

[15] Cox, J., Ingersoll, J., and S., Ross (1985): "A Theory of the Term Structure of Interest Rates”, Econometrica, 53, 385-407.

[16] Darolles, S., Gourieroux, C., and J., Jasiak (2006): ”Structural Laplace Transform and Compound Autoregressive Models", Journal of Time Series Analysis, 27, 477-503.

[17] Davis, M., and V., Lo (2001)a: "Infectious Defaults", Quantitative Finance, 1, 382-387.

[18] Davis, M., and V., Lo (2001)b: "Modelling Default Correlation in Bond Portfolios", in Alexander, C. (ed), Mastering Risk, Vol 2: Applications. Financial Times, Prentice Hall, Englewood Cliffs, NJ, 141-151.

[19] de Finetti, B. (1931): "Funzione Caratteristica di un Fenomeno Aleatorio", Atti della R. Academia dei Lincei, Memorie, 6, Classe di Scienze Fisiche, Mathematiche e Naturali, 4, 251-299.

[20] Delloy, M., Fermanian, J. D., and M., Sbai (2005): "Estimation of a Reduced Form Credit Portfolio Model and Extensions to Dynamic Frailties", DP BNP Paribas.

[21] Duffie, D., Eckner, A., Horel, G., and L., Saita (2009): "Frailty Correlated Default", Journal of Finance, 64, 2089-2183.

[22] Duffie, D., Filipovic, D., and W., Schachermayer (2003): "Affine Processes and Applications in Finance", Annals of Applied Probability, 13, 984-1053.

[23] Duffie, D., and K., Singleton (1998): “Simulating Correlated Defaults”, Working Paper, Stanford University.

[24] Egloff, D., Leippold, M., and P., Vanini (2007): "A Simple Model of Credit Contagion", Journal of Banking and Finance, 31, 2475-2492. 
[25] Feller, W. (1968): An Introduction to Probability Theory and its Applications, Wiley.

[26] Fournier, N., and S., Meleard (2004): "A Microscopic Probabilistic Description of a Locally Regulated Population and Macroscopic Approximations“, The Annals of Applied Probability, 14, 1880-1919.

[27] Freund, J. (1961): "A Bivariate Extension of the Exponential Distribution“, Journal of the American Statistical Society, 56, 971-977.

[28] Frey, R., and J., Backhaus (2003): ”Interacting Defaults and Counterparty Risk: a Markovian Approach”, DP University of Leipzig.

[29] Frey, F., and J., McNeil (2003): "Modeling Dependent Defaults", Journal of Risk, 6, 59-92.

[30] Gagliardini, P., Gourieroux, C., and A., Monfort (2012): "Microinformation, Nonlinear Filtering and Granularity", Journal of Financial Econometrics, 10, 1-53.

[31] Gallo, G., and E., Otranto (2007): "Volatility Transmission Across Markets: A Multichain Markov Switching Model“, Applied Financial Economics, 17, 659-670.

[32] Giesecke, K. (2004): "Correlated Default with Incomplete Information”, Journal of Banking and Finance, 28, 1521-1545.

[33] Giesecke, K., and S., Weber (2004): "Cyclical Correlation, Credit Contagion and Portfolio Losses”, Journal of Banking and Finance, 28, 1521-1545.

[34] Giesecke, K., and S., Weber (2006): "Credit Contagion and Aggregate Losses”, Journal of Economic Dynamics and Control, 30, 741-767.

[35] Gordy, M. (2003): "A Risk-Factor Model Foundation for Rating-Based Bank Capital Rules", Journal of Financial Intermediation, 12, 199-232.

[36] Gordy, M. (2004): "Granularity Adjustment in Portfolio CreditRisk Measurement", in G. Szego, ed., Risk Measures for the 21.st Century, Wiley.

[37] Gourieroux, C., Heam, J. C., and A., Monfort (2011): ”Equilibrium and Contagion of Default Risks in a Banking System", CREST DP. 
[38] Gourieroux, C., and J., Jasiak (2001): ”Dynamic Factor Models”, Econometric Reviews, 20, $385-424$.

[39] Gourieroux, C., and J., Jasiak (2004): "Heterogenous INAR(1) Model with Application to Car Insurance”, Insurance: Mathematics and Economics, 34, 177-192.

[40] Gourieroux, C., and J., Jasiak (2006): "Autoregressive Gamma Processes", International Journal of Forecasting, 25, 129-152.

[41] Granger, C., and A., Andersen (1978): "Nonlinear Time Series Modelling“, in Applied Time Series Analysis, D. Finley ed., Academic Press, New York, 25-38.

[42] Greenwood, M., and G., Yule (1920): ”An Inquiry Into the Nature of Frequency Distribution Representation of Multiple Happenings with Particular Reference to the Occurrence of Multiple Attacks of Disease or of Repeated Accidents", Journal of the Royal Statistical Society, $83,255-279$.

[43] Gschlossl, S., and C., Czado (2006): "Modelling Count Data with Overdispersion and Spatial Effects“, Munich University DP 475.

[44] Hawkes, A. (1971)a: "Spectra of Some Self-Exciting and Mutually Exciting Point Processes", Biometrika, 58, 83-90.

[45] Hawkes, A. (1971)b: "Point Spectra of Some Mutually Exciting Point Processes", Journal of the Royal Statistical Society B, 33, 438-443.

[46] Hawkes, A., and D., Oakes (1974): "A Cluster Representation of a Self-Exciting Process", Journal of Applied Probability, 11, 493-503.

[47] Hewitt, E., and L., Savage (1955): "Symmetric Measures on Cartesian Products", Transaction of the American Mathematical Society, 80, 470-501.

[48] Jarrow, R., and F., Yu (2001): "Counterparty Risk and the Pricing of Defaultable Securities", Journal of Finance, 56, 1765-1800. 
[49] Kendall, D. (1956): "Deterministic and Stochastic Epidemics in Closed Populations“, Proceedings of the 3rd Berkeley Symposium on Mathematical Statistics and Probability, Vol. IV, 149-165.

[50] Lando, D., and M., Nielsen (2010): "Correlation in Corporate Defaults: Contagion or Conditional Independence?”, Journal of Financial Intermediation, 355-372.

[51] Li, D. (2000): "On Default Correlation: A Copula Function Approach", Journal of Fixed Income, 9, 43-54.

[52] Manski, C. (1993): "Identification of Endogenous Social Effects: the Reflection Problem“, Review of Economic Studies, 60, 531-542.

[53] McKenzie, E. (1985): "Some Simple Models for Discrete Variate Time Series", Water Resources Bulletin, 21, 645-650.

[54] McKenzie, E. (1988): "Some ARMA Models for Dependent Sequences of Poisson Counts", Advances in Applied Probability, 20, 822-835.

[55] Meyn, S., and R., Tweedie (2009): Markov Chains and Stochastic Stability, Second Edition, Cambridge University Press.

[56] Pham, T., and T., Tran (1981): ”On the First Order Bilinear Time Series Model“, Journal of Applied Probability, 18, 617-627.

[57] Rosch, D., and B., Winterfeld (2008): "Estimating Credit Contagion in a Standard Factor Model“", Risk, August, 78-82.

[58] Rulliere, D., Dorobantu, D., and A., Cousin (2010): ”An Extension of Davis and Lo's Contagion Model“", Lyon University DP.

[59] Sakata, A., Hisakado, M., and S., Mori (2007): 'Infectious Default Model with Recovery and Continuous Limits", Journal of the Physical Society of Japan, 76, 5.

[60] Schoenbucher, P. (2001): "Factor Models for Portfolio Credit Risk When Defaults are Correlated", The Journal of Risk Finance, 3, 45-56. 
[61] Schonbucher, P., and D., Schubert (2001): "Copula-Dependent Default Risk in Intensity Models", DP Bonn University.

[62] Steutel, F., and K., Van Harn (1979): "Discrete Analogues of Self-Decomposability and Stability", The Annals of Probability, 7, 893-899.

[63] Upper, C., and A., Worms (2004): "Estimating Bilateral Exposures in the German Interbank Market: Is There a Danger of Contagion?”, European Economic Review, 48, 827-849.

[64] Vasicek, O. (1987): "The Loan Loss Distribution", DP KMV Corporation.

[65] Vaupel, J., Manton, K., and E., Stallard (1979): "The Impact of Heterogeneity in Individual Frailty on the Dynamics of Mortality", Demography, 16, 439-454. 
Figure 1: Equilibrium value and autoregressive coefficient in the logistic model.
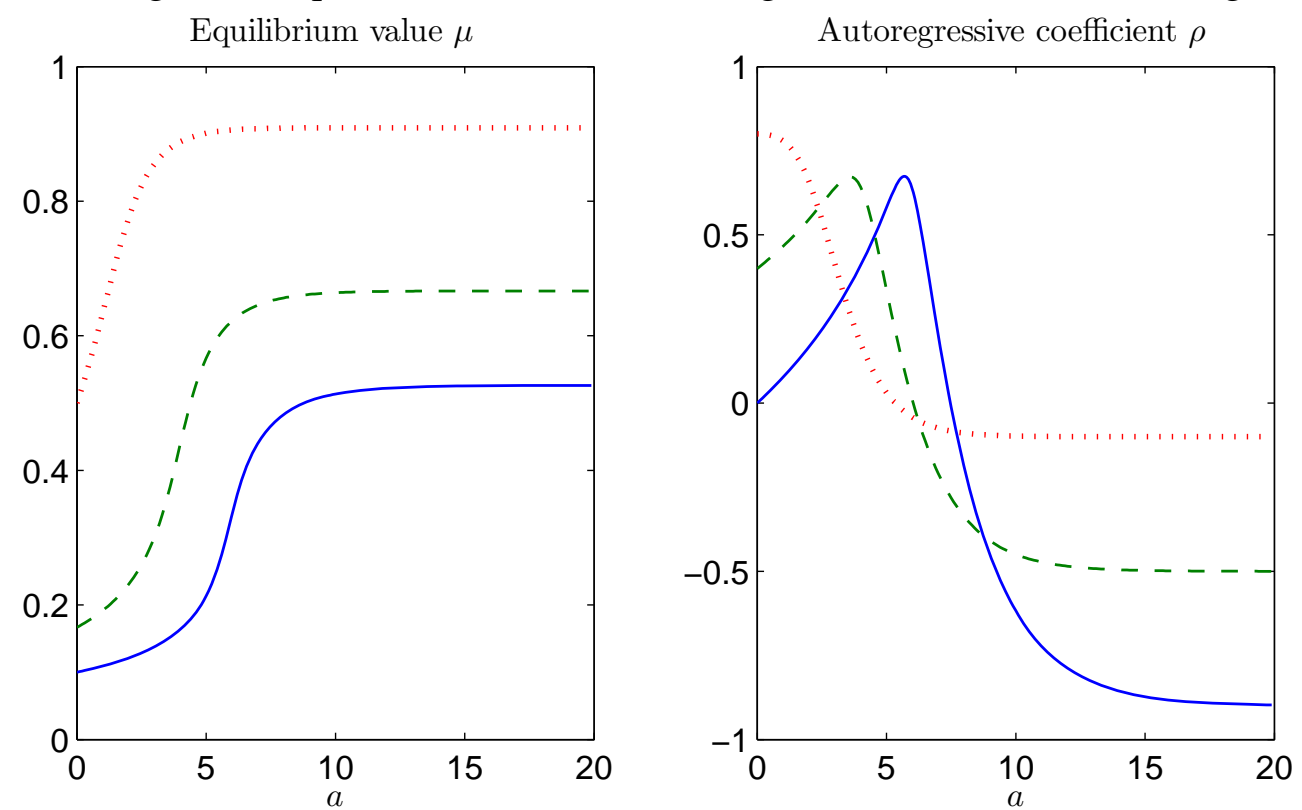

The left panel displays the equilibrium value $\mu$ as a function of parameter $a$, while the right panel displays the autoregressive coefficient $\rho^{*}$ as a function of $a$, for the logistic model with contagion in equation (3.12). In each panel, the three curves correspond to different values of parameter $p_{11}$, that are $p_{11}=1 / 10$ (solid line), $p_{11}=1 / 2$ (dashed line) and $p_{11}=9 / 10$ (dotted line). Parameter $b$ is equal to $b=-\log (9)$. 
Figure 2: Simulated paths of factor, count and stochastic equilibrium in the logistic model with contagion and correlated risks.

Factor $F_{t}$

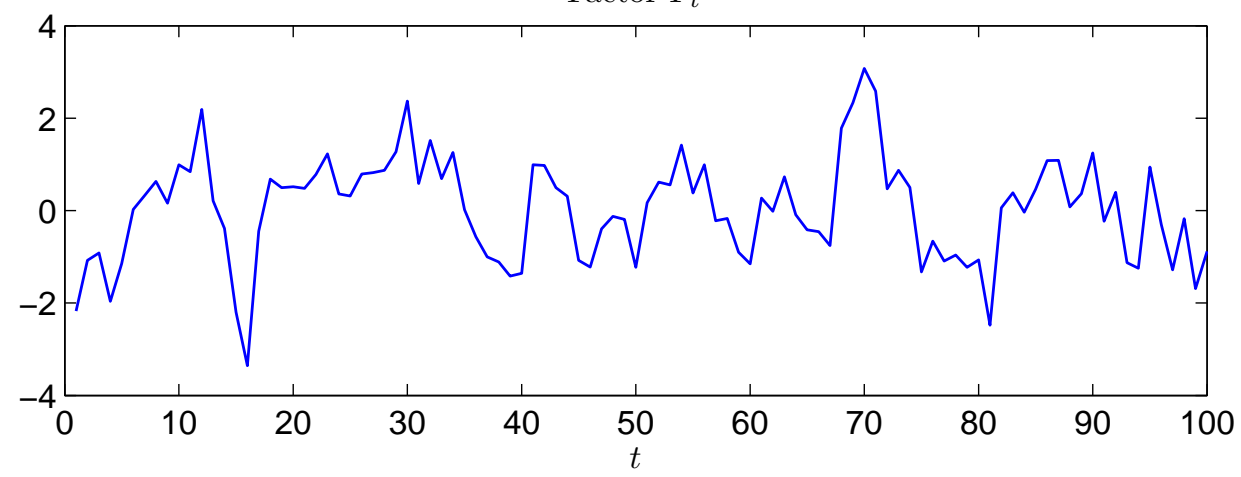

Fraction $N_{t} / n$

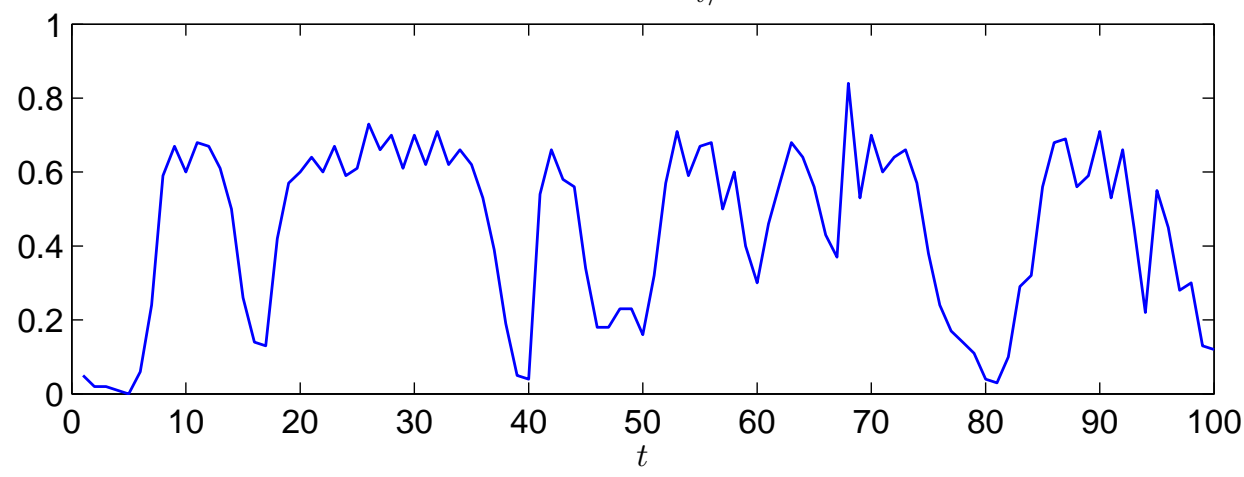

Equilibrium $\mu_{t}$

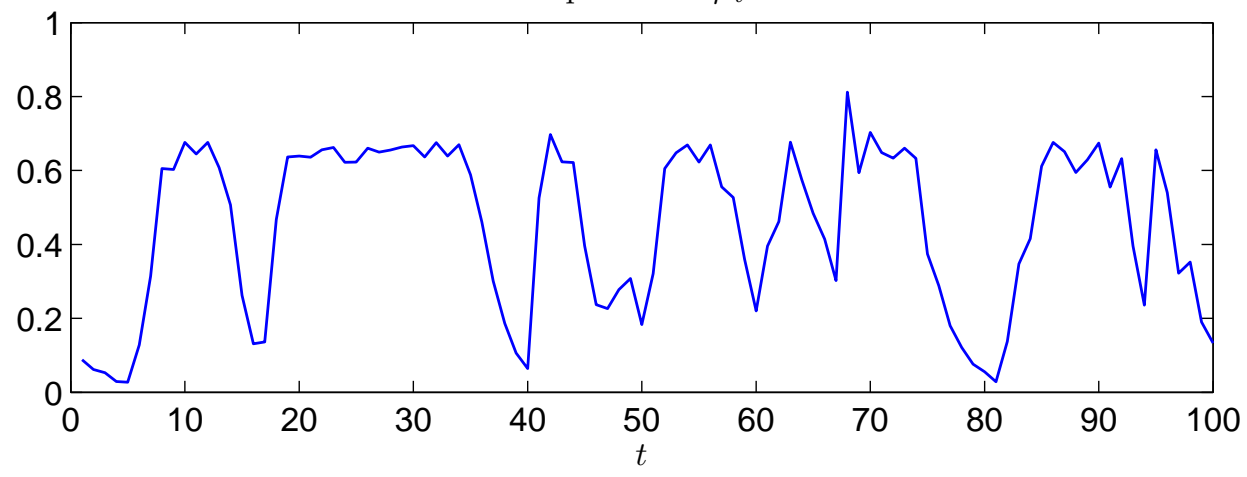

This Figure displays simulated paths for the logistic model with contagion and correlated risks (3.17)-(3.18). The parameter values are $a=5, b=-\log (9), c=2, p_{11}=0.5$ and $\gamma=0.5$. The number of individuals is $n=100$. The upper Panel displays the path of the factor $F_{t}$, the middle Panel displays the path of the fraction $N_{t} / n$ of individuals in state 1 , and the lower Panel displays the path of the stochastic equilibrium $\mu_{t}$. 
Figure 3: Scatter plot and support of the stationary distribution of the joint equilibrium and factor process for the logistic model with contagion and correlated risks.

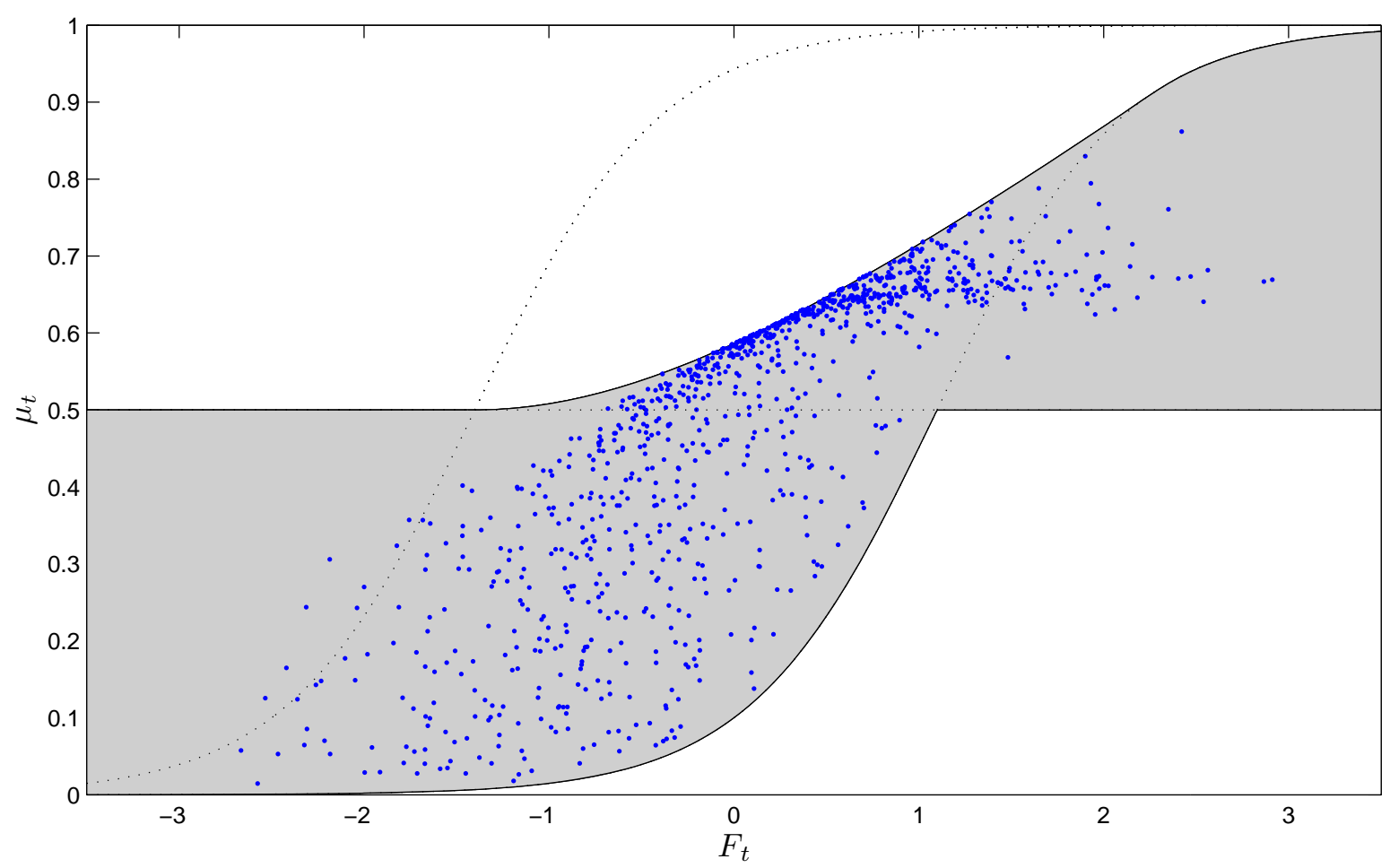

This Figure displays the scatter plot of a simulated sample of the joint process $\left(\mu_{t}, F_{t}\right)$ for the logistic model with contagion and correlated risks (3.17)-(3.18). The parameter values are $a=5, b=-\log (9), c=2, p_{11}=0.5$ and $\gamma=0.5$. The shaded area is the support of the stationary distribution of $\left(\mu_{t}, F_{t}\right)$, that corresponds to set $\Omega$ in equation (a.6) in Appendix A.2.1. The horizontal dashed line corresponds to $\mu=p_{11}$. The two dashed curves correspond to functions $\mu=p_{10}(F, 0)$ (lower curve) and $\mu=p_{10}(F, 1)$ (upper curve). 
Figure 4: Simulated paths of deviation from equilibrium, autoregressive coefficient and volatility of the Gaussian approximation for the logistic model with contagion and correlated risks.

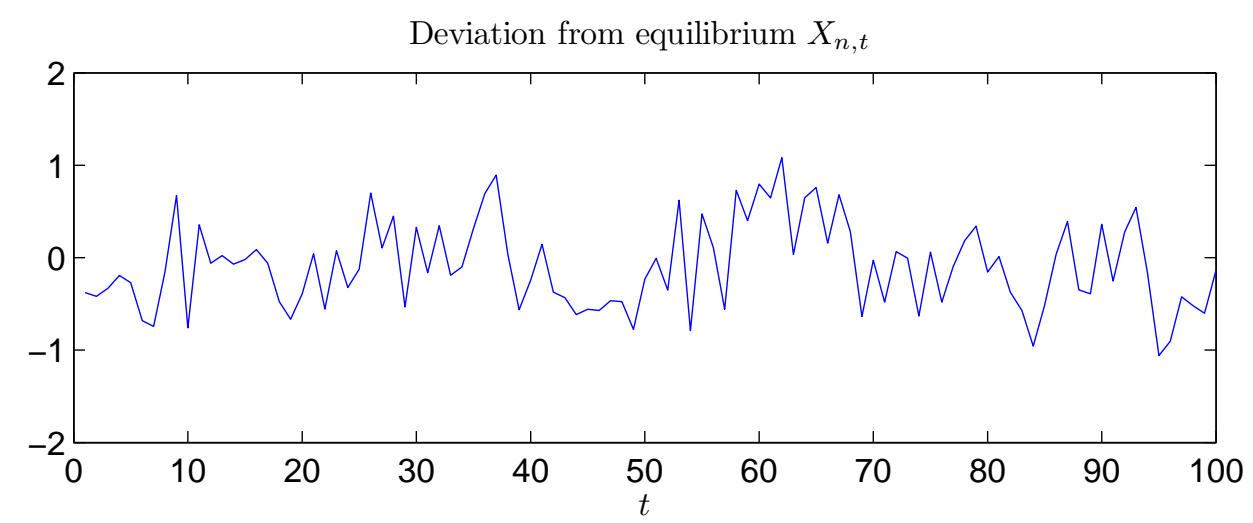

Autocorrelation coefficient $\rho_{t}^{*}$

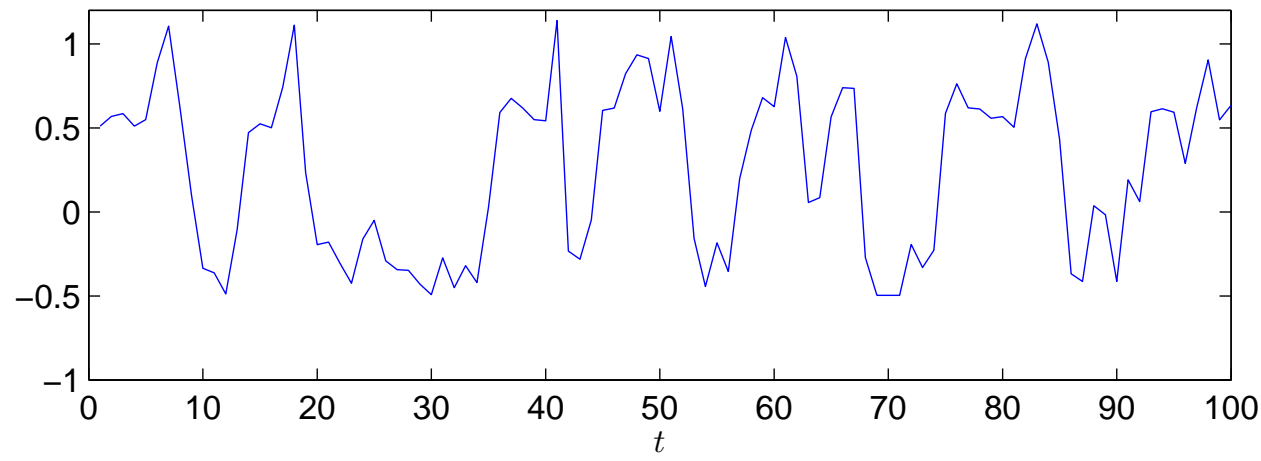

Volatility parameter $\eta_{t}^{*}$

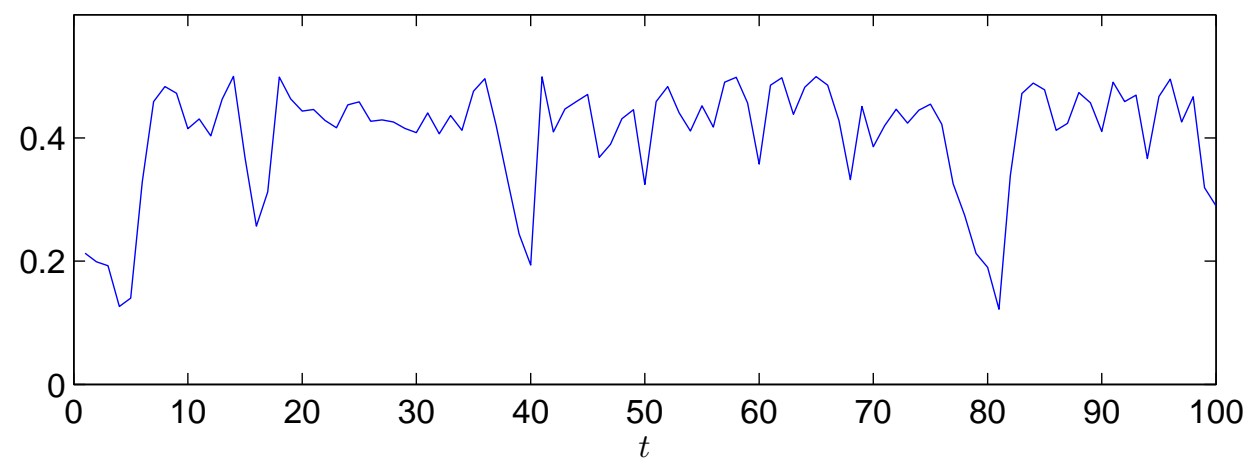

This Figure displays simulated paths for the logistic model with contagion and correlated risks (3.17)-(3.18). The parameter values are $a=5, b=-\log (9), c=2, p_{11}=0.5$ and $\gamma=0.5$. The number of individuals is $n=100$. The upper Panel displays the path of the standardized deviation from equilibrium $X_{n, t}=\sqrt{n}\left(N_{t} / n-\mu_{t}\right)$, the middle Panel displays the path of the autocorrelation coefficient $\rho_{t}^{*}$ of the Gaussian approximation $\xi_{t}^{*}$ in Corollary 2 , and the lower Panel displays the path of its volatility parameter $\eta_{t}^{*}$. 
Figure 5: Simulated paths of the INAR process with stochastic intensity (parameter sets A and B).
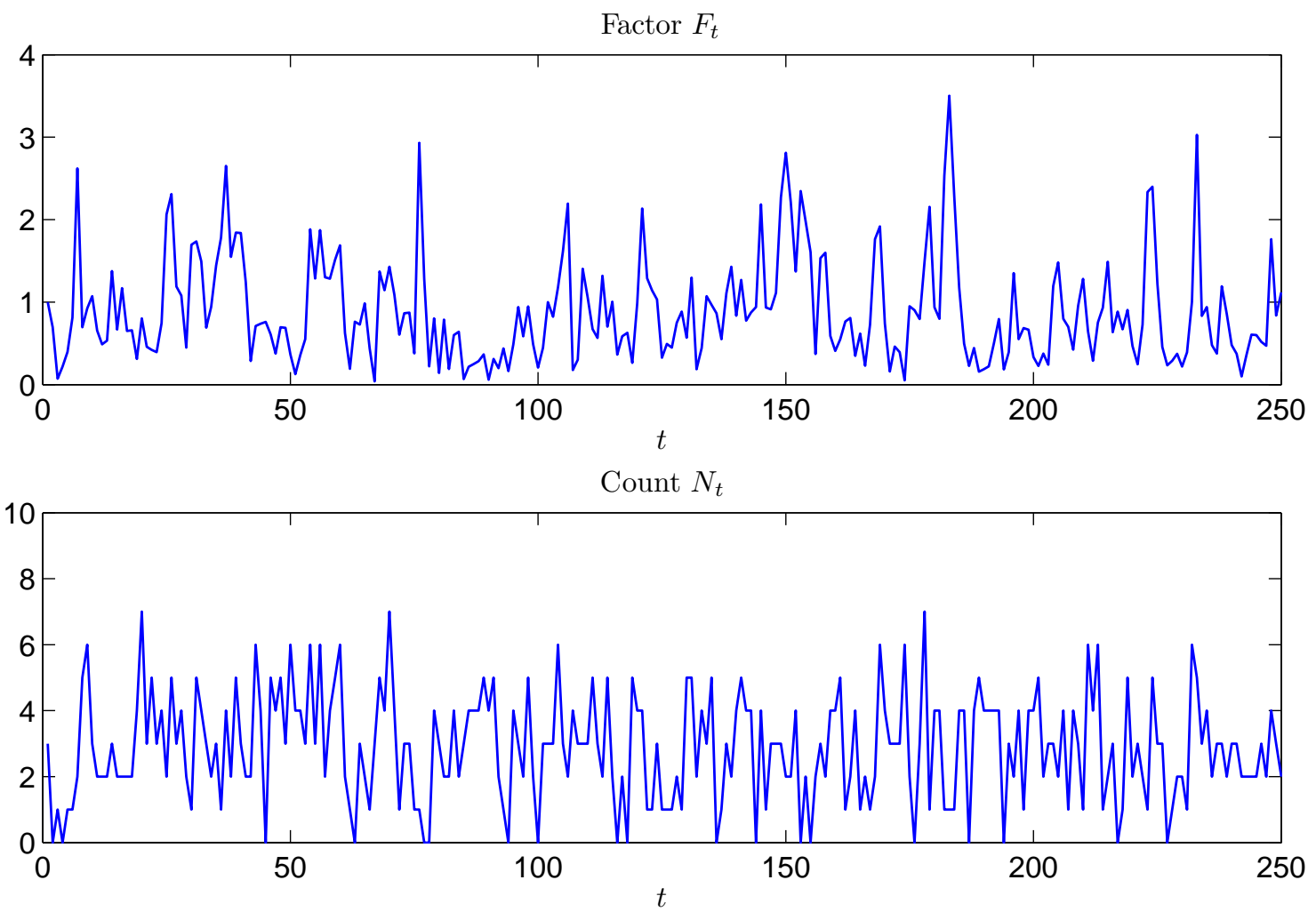

Count $N_{t}$

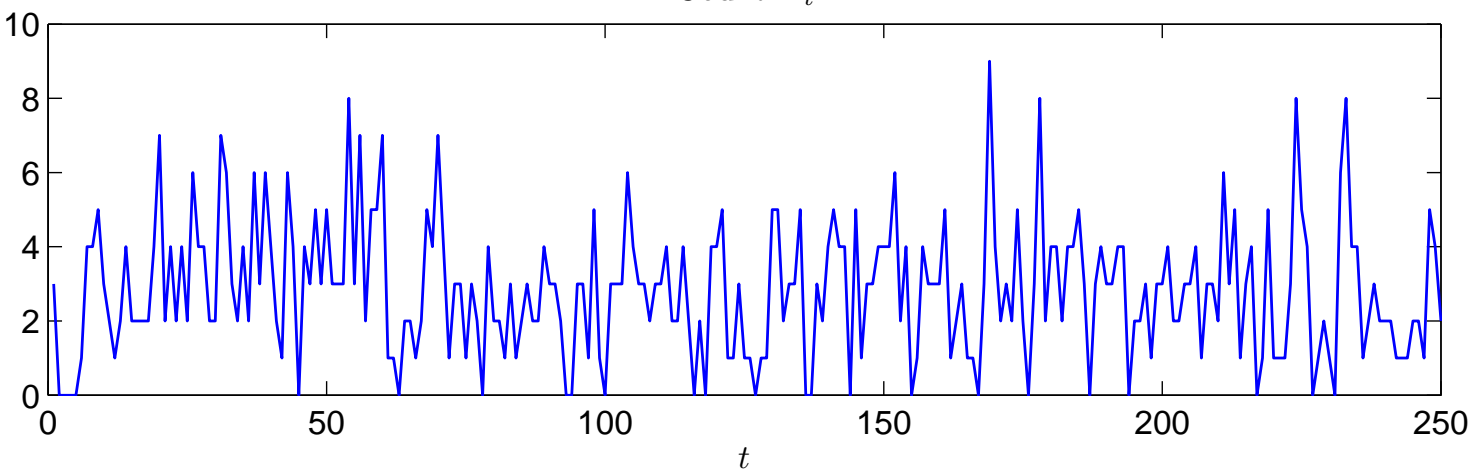

This Figure displays simulated paths for the INAR process with stochastic intensity as in Assumptions A.5-A.6. The factor $F_{t}$ follows an ARG process with parameters $\gamma=0.5, \delta=2$ and $\eta=0.25$. The autoregressive parameter $\rho$ of the INAR model is $\rho=0.2$. The upper panel displays the factor path. The middle and lower panels display the paths of the count $N_{t}$ for models with intensity parameters $c_{0}, c_{1}$ and $c_{2}$ as in sets A and B, respectively. 
Figure 6: Simulated paths of the INAR process with stochastic intensity (parameter sets C and D).

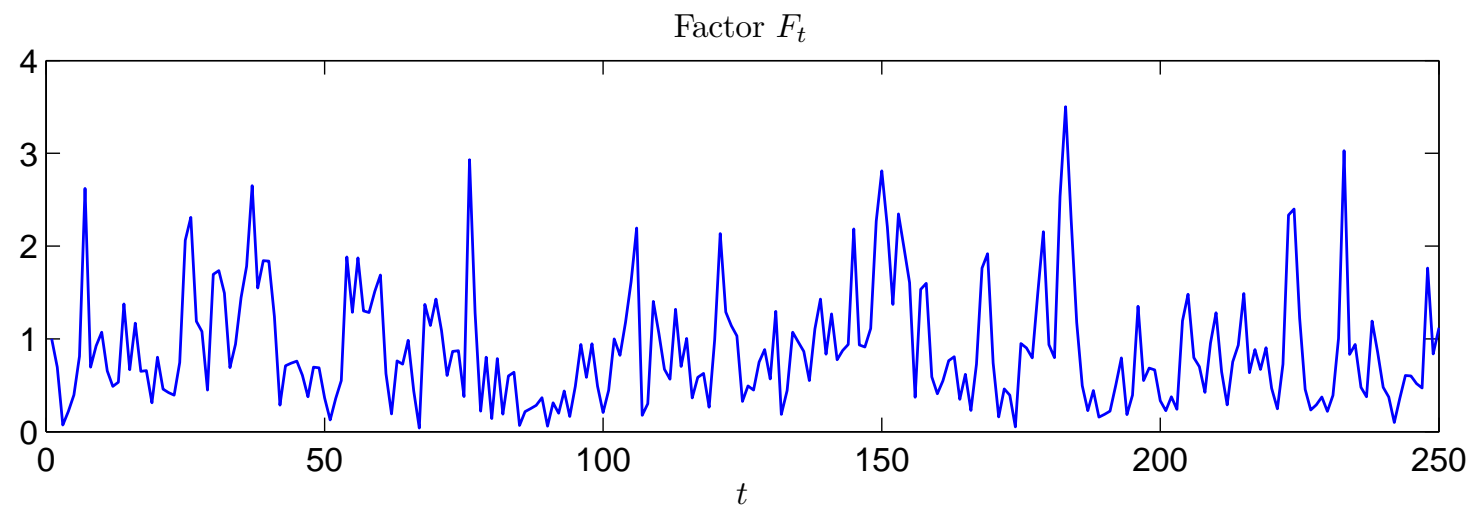

Count $N_{t}$

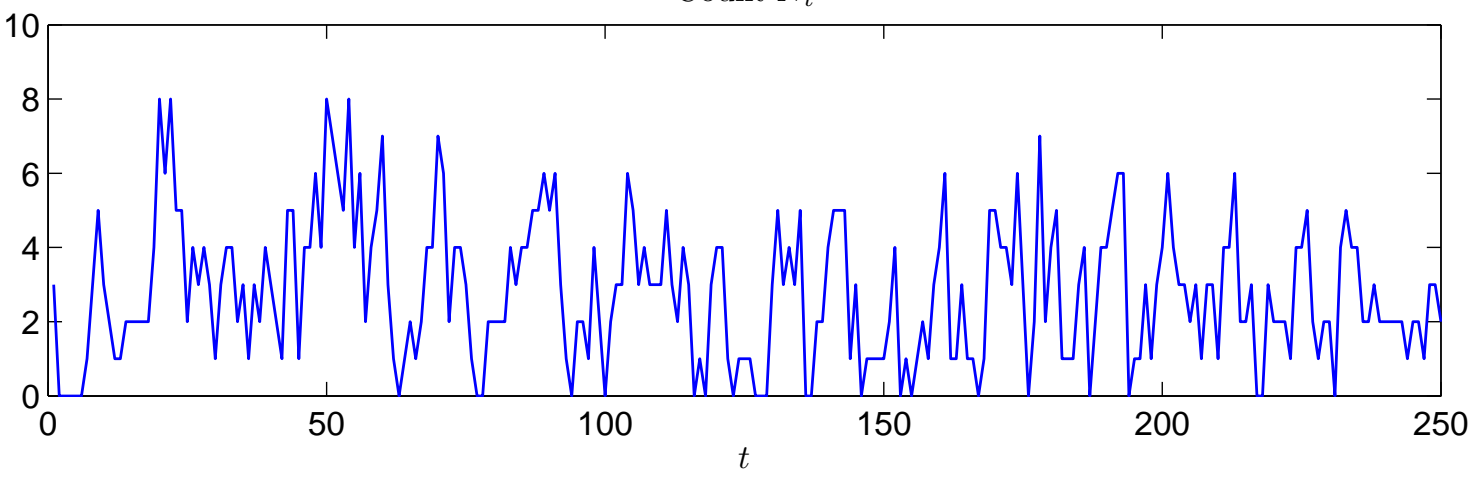

Count $N_{t}$

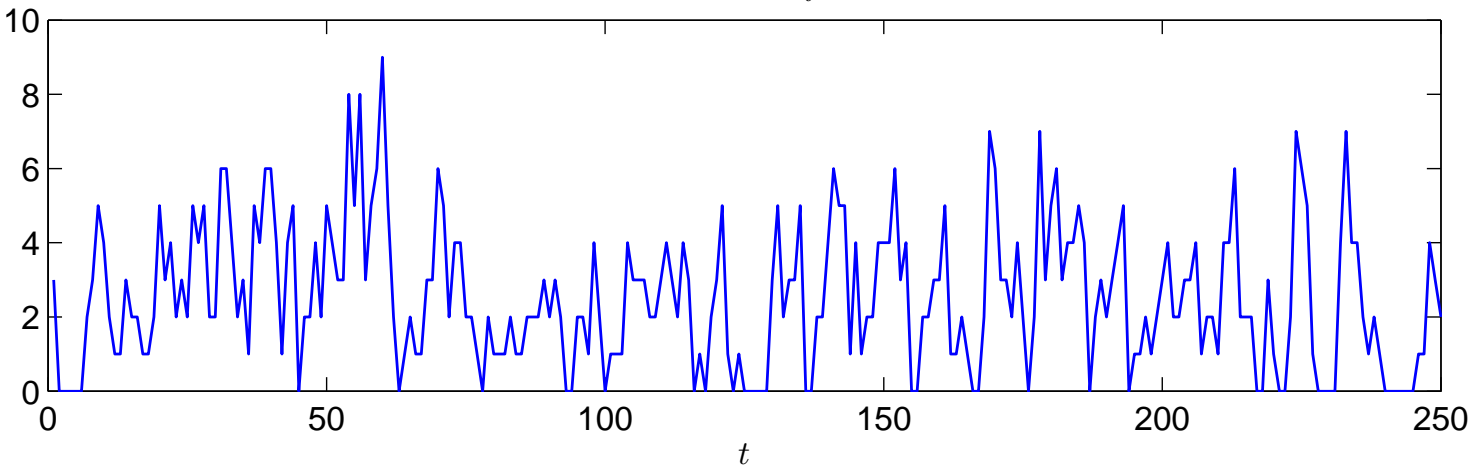

This Figure displays simulated paths for the INAR process with stochastic intensity as in Assumptions A.5-A.6. The factor $F_{t}$ follows an ARG process with parameters $\gamma=0.5, \delta=2$ and $\eta=0.25$. The autoregressive parameter $\rho$ of the INAR model is $\rho=0.2$. The upper panel displays the factor path. The middle and lower panels display the paths of the count $N_{t}$ for models with intensity parameters $c_{0}, c_{1}$ and $c_{2}$ as in sets $\mathrm{C}$ and $\mathrm{D}$, respectively. 
Figure 7: Conditional expectation of $N_{t}$ given $N_{t-1}$ in the INAR process with stochastic intensity.

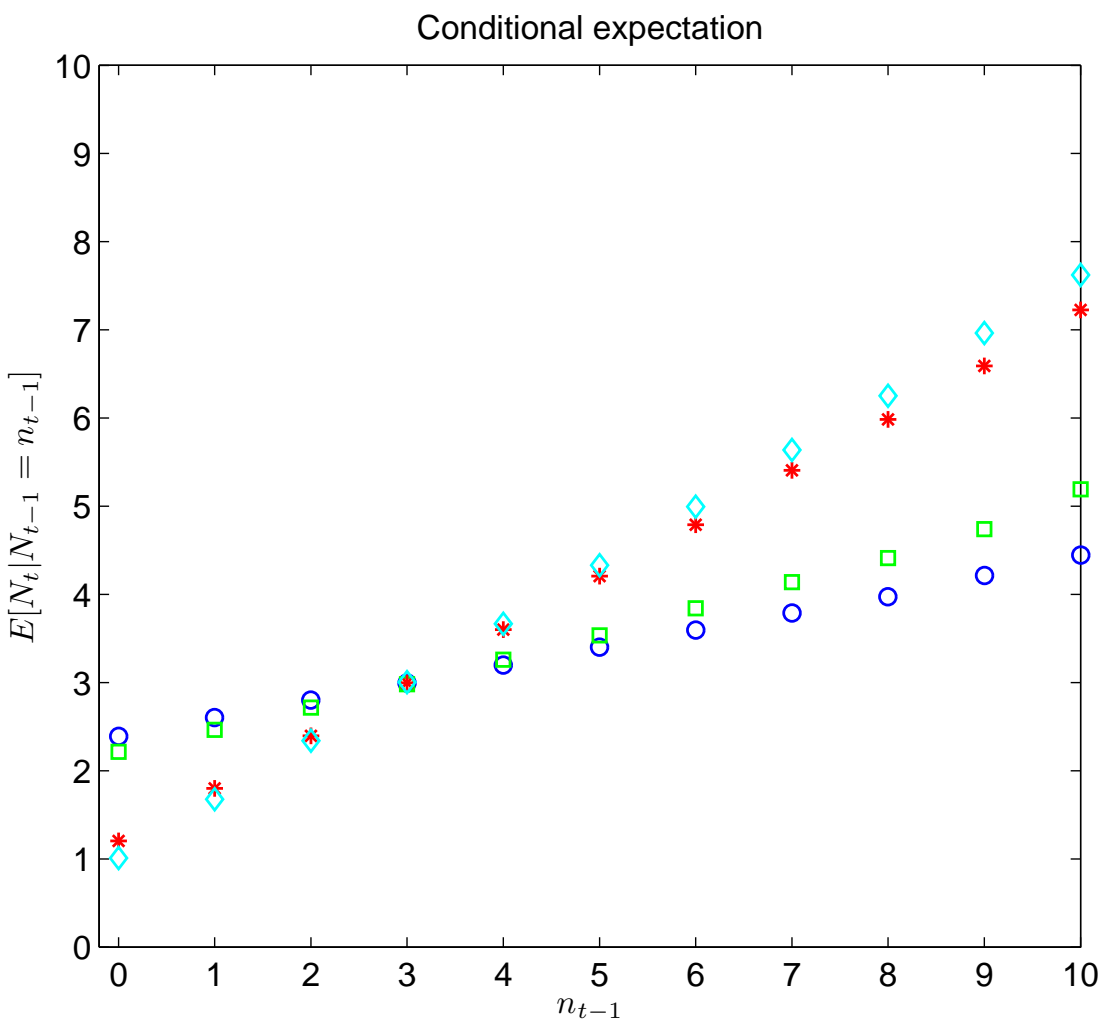

This Figure displays the conditional expectation of $N_{t}$ given $N_{t-1}$ for the INAR process with stochastic intensity as in Assumptions A.5-A.6. The factor $F_{t}$ follows an ARG process with parameters $\gamma=0.5, \delta=2$ and $\eta=0.25$. The autoregressive parameter $\rho$ of the INAR model is $\rho=0.2$. Circles, squares, stars and diamonds correspond to intensity parameters $c_{0}, c_{1}$ and $c_{2}$ as in sets $\mathrm{A}, \mathrm{B}, \mathrm{C}$ and $\mathrm{D}$, respectively. 
Figure 8: Autocorrelogram of the INAR process $\left(N_{t}\right)$ with stochastic intensity.
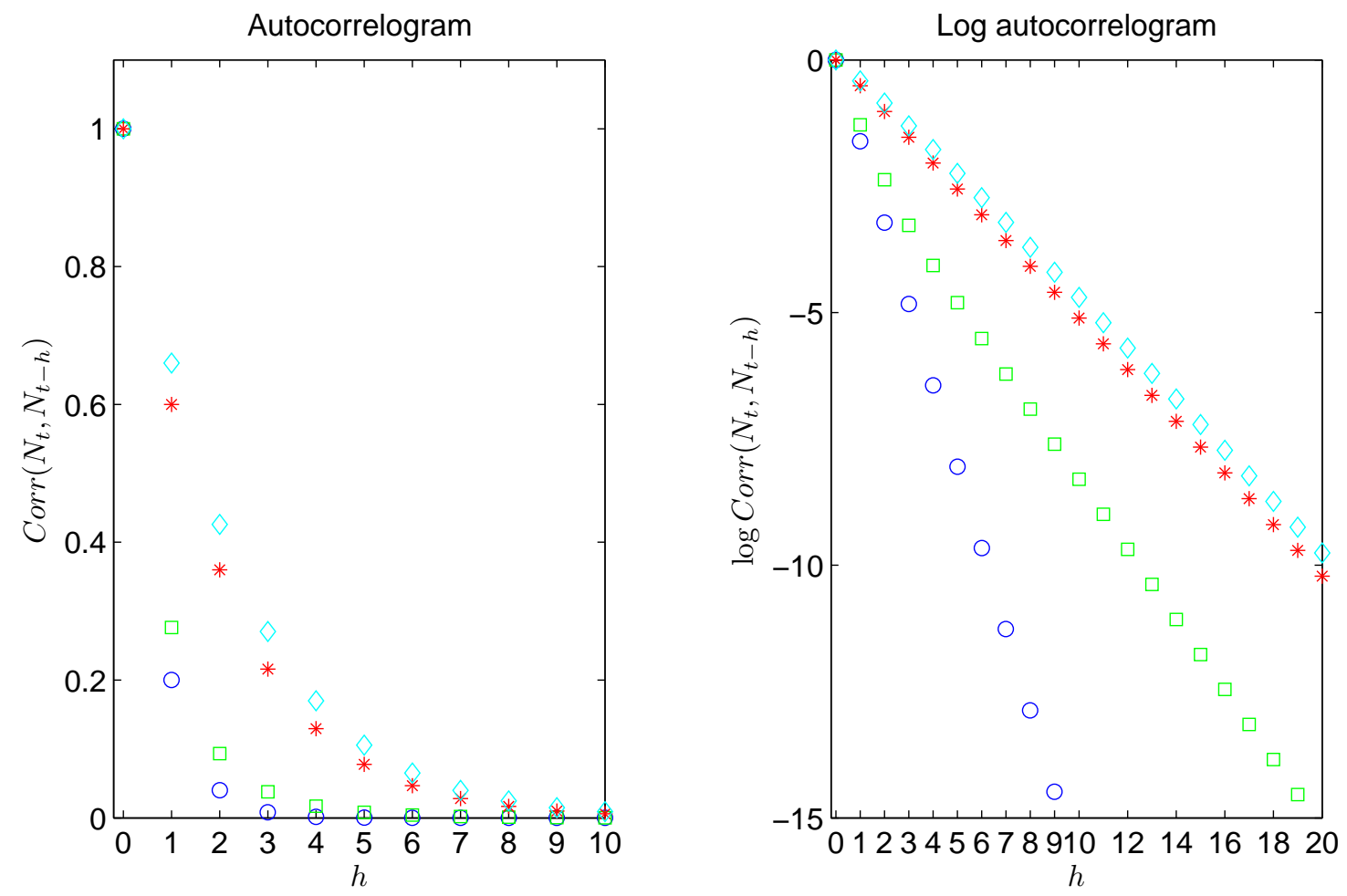

This Figure displays the autocorrelogram (left panel) and the log autocorrelogram (right panel) of the INAR process $\left(N_{t}\right)$ with stochastic intensity as in Assumptions A.5-A.6. The factor $F_{t}$ follows an ARG process with parameters $\gamma=0.5, \delta=2$ and $\eta=0.25$. The autoregressive parameter $\rho$ of the INAR model is $\rho=0.2$. Circles, squares, stars and diamonds correspond to intensity parameters $c_{0}, c_{1}$ and $c_{2}$ as in sets $\mathrm{A}, \mathrm{B}, \mathrm{C}$ and $\mathrm{D}$, respectively. 
Figure 9: Conditional overdispersion function of the INAR process $\left(N_{t}\right)$ with stochastic intensity.

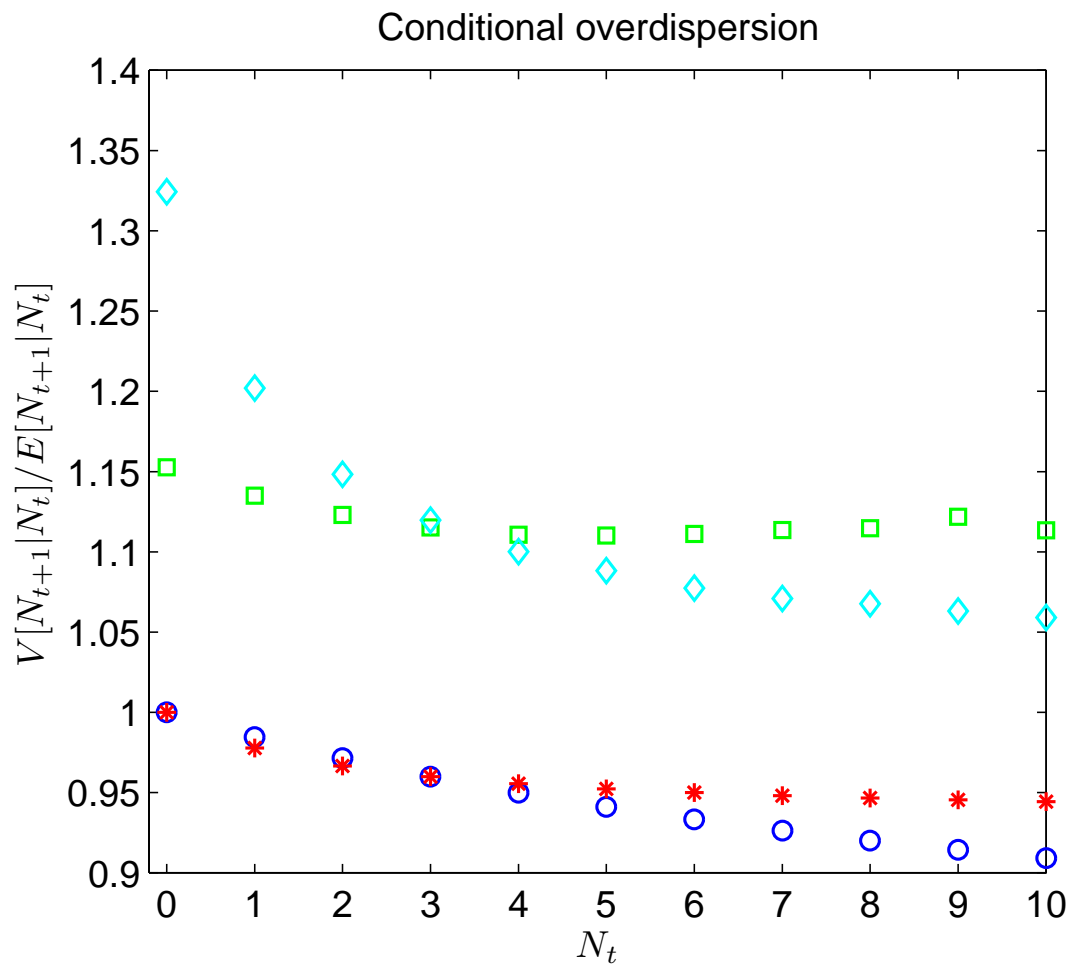

This Figure displays the pattern of the conditional overdispersion of $N_{t+1}$ given $N_{t}$, as a funtion of $N_{t}$, for the INAR process $\left(N_{t}\right)$ with stochastic intensity as in Assumptions A.5-A.6. The factor $F_{t}$ follows an ARG process with parameters $\gamma=0.5, \delta=2$ and $\eta=0.25$. The autoregressive parameter $\rho$ of the INAR model is $\rho=0.2$. Circles, squares, stars and diamonds correspond to intensity parameters $c_{0}, c_{1}$ and $c_{2}$ as in sets $\mathrm{A}, \mathrm{B}, \mathrm{C}$ and $\mathrm{D}$, respectively. 
Table 1: Summary statistics and autocorrelogram of process $\tilde{\varepsilon}_{t}^{*}$.

\begin{tabular}{lccc}
\hline \hline & $n=25$ & $n=100$ & $n=1000$ \\
Mean & -0.068 & -0.035 & -0.011 \\
Median & -0.076 & -0.041 & -0.012 \\
Std. deviation & 1.001 & 1.001 & 1.000 \\
Skewness & 0.015 & 0.025 & 0.013 \\
Kurtosis & 3.156 & 3.042 & 3.000 \\
\hline$A C(1)$ & 0.016 & 0.006 & 0.000 \\
$A C(2)$ & 0.002 & 0.002 & 0.001 \\
$A C(3)$ & 0.002 & -0.000 & 0.001 \\
$A C(4)$ & 0.001 & 0.001 & 0.000 \\
$A C(5)$ & 0.000 & 0.000 & 0.000 \\
\hline \hline
\end{tabular}

This Table displays summary statistics and the autocorrelogram of process $\tilde{\varepsilon}_{t}^{*}=\frac{X_{n, t}-\rho_{t}^{*} X_{n, t-1}}{\eta_{t}^{*}}$ for the logistic model with contagion and correlated risks (3.17)-(3.18). The parameter values are $a=5$, $b=-\log (9), c=2, p_{11}=0.5$ and $\gamma=0.5$. We consider different population sizes that are $n=25, n=100$ and $n=1000$. Statistic $A C(h)=\operatorname{Corr}\left(\tilde{\varepsilon}_{t}^{*}, \tilde{\varepsilon}_{t-h}^{*}\right)$ denotes the autocorrelation of $\tilde{\varepsilon}_{t}^{*}$ of order $h$. 


\section{APPENDIX 1: Proofs of Proposition 2 and Corollary 1}

\section{A.1.1 Proof of Proposition 2}

Let $\left(X_{n, t}\right) \Rightarrow\left(\xi_{t}\right)$ denote convergence in distribution (or weak convergence) of the sequence of processes $\left(X_{n, t}=\sqrt{n}\left[N_{t} / n-\mu\right]: t \in \mathbb{N}\right), n=1,2, \ldots$ with sample space $\mathbb{R}^{\infty}$ to process $\left(\xi_{t}: t \in \mathbb{N}\right)$ when $n \rightarrow \infty$. From Billingsley (1968), p. $19,\left(X_{n, t}\right) \Rightarrow\left(\xi_{t}\right)$ is equivalent to convergence of the finite-dimensional distributions, i.e.:

$$
P\left[X_{n, 1} \in A_{1}, \ldots, X_{n, t} \in A_{t}\right] \rightarrow P\left[\xi_{1} \in A_{1}, \ldots, \xi_{t} \in A_{t}\right], \quad n \rightarrow \infty
$$

for any set $A_{\tau}$ in the Borel sigma-field $\mathcal{B}(\mathbb{R})$ with $P\left[\xi_{\tau} \in \partial A_{\tau}\right]=0$, for $\tau=1, \ldots, t$, and $t \in \mathbb{N}$. Now, we use that $\left(X_{n, t}\right)$ and $\left(\xi_{t}\right)$ are Markov processes, and that the convergence of a sequence of finite-dimensional distributions can be written in terms of the convergence of the sequence of the associated characteristic functions. We get the next Lemma, which shows that condition (a.1) holds if we have the convergence of the initial distribution and of the conditional characteristic function of process $\left(X_{n, t}\right)$, as well as the continuity of the conditional characteristic function of the limit process $\left(\xi_{t}\right)$ w.r.t. the conditioning variable.

\section{Lemma A.1: Suppose that:}

(i) $X_{n, 1}$ converges in distribution to $\xi_{1}$.

(ii) $\limsup _{n \rightarrow \infty} \int\left|E\left[e^{i u X_{n, t}} \mid X_{n, t-1}=x\right]-E\left[e^{i u \xi_{t}} \mid \xi_{t-1}=x\right]\right| \nu_{n, t-1}(d x)=0$, for any $u \in \mathbb{R}$ and $t=$ $2,3, \ldots$, where $\nu_{n, t-1}$ denotes the probability measure of $X_{n, t-1}$ and $i$ is the imaginary unit.

(iii) The function $x \rightarrow E\left[e^{i u \xi_{t}} \mid \xi_{t-1}=x\right]$ is continuous, for any $u \in \mathbb{R}$ and $t \in \mathbb{N}$.

Then, condition (a.1) holds.

Proof of Lemma A.1: For any $t \in \mathbb{N}$ let $\nu_{n}^{(t)}$ and $\nu^{(t)}$ denote the probability measures of the random vectors $\left(X_{n, 1}, \ldots, X_{n, t}\right)^{\prime}$ and $\left(\xi_{1}, \ldots, \xi_{t}\right)^{\prime}$, respectively. Then, condition (a.1) is equivalent to the weak convergence $\nu_{n}^{(t)} \Rightarrow \nu^{(t)}$, for any $t \in \mathbb{N}$, as well as to:

$$
E\left[e^{i u_{1} X_{n, 1}+\ldots+i u_{t} X_{n, t}}\right] \rightarrow E\left[e^{i u_{1} \xi_{1}+\ldots+i u_{t} \xi_{t}}\right], \quad n \rightarrow \infty
$$

for any $u_{1}, \ldots, u_{t} \in \mathbb{R}$ and $t \in \mathbb{N}$ [see Billingsley (1968), Theorems 1.2.1 and 1.7.6]. We prove convergence (a.1) by induction in $t \in \mathbb{N}$. For $t=1$, convergence (a.1) is implied by condition (i). 
Let us now assume that convergence (a.1) holds for a given integer $t-1$, and prove that it is valid for $t$ as well. By the Markov property of process $\left(X_{n, t}\right)$ we have:

$$
\begin{aligned}
E\left[e^{i u_{1} X_{n, 1}+\ldots+i u_{t} X_{n, t}}\right] & =E\left[E\left[e^{i u_{t} X_{n, t}} \mid X_{n, t-1}\right] e^{i u_{1} X_{n, 1}+\ldots+i u_{t-1} X_{n, t-1}}\right] \\
& =\int E\left[e^{i u_{t} X_{n, t}} \mid X_{n, t-1}=x_{t-1}\right] e^{i u_{1} x_{1}+\ldots+i u_{t-1} x_{t-1}} \nu_{n}^{(t-1)}\left(d x^{(t-1)}\right),
\end{aligned}
$$

where $x^{(t-1)}:=\left(x_{1}, \ldots, x_{t-1}\right) \in \mathbb{R}^{t-1}$. Similarly, by the Markov property of process $\left(\xi_{t}\right)$, we have:

$$
E\left[e^{i u_{1} \xi_{1}+\ldots+i u_{t} \xi_{t}}\right]=\int E\left[e^{i u_{t} \xi_{t}} \mid \xi_{t-1}=x_{t-1}\right] e^{i u_{1} x_{1}+\ldots+i u_{t-1} x_{t-1}} \nu^{(t-1)}\left(d x^{(t-1)}\right) .
$$

Thus, we get:

$$
\begin{aligned}
\left|E\left[e^{i u_{1} X_{n, 1}+\ldots+i u_{t} X_{n, t}}\right]-E\left[e^{i u_{1} \xi_{1}+\ldots+i u_{t} \xi_{t}}\right]\right| \leq \int\left|E\left[e^{i u_{t} X_{n, t}} \mid X_{n, t-1}=x\right]-E\left[e^{i u_{t} \xi_{t}} \mid \xi_{t-1}=x\right]\right| \nu_{n, t-1}(d x) \\
+\int E\left[e^{i u_{t} \xi_{t}} \mid \xi_{t-1}=x_{t-1}\right] e^{i u_{1} x_{1}+\ldots+i u_{t-1} x_{t-1}}\left[\nu_{n}^{(t-1)}-\nu^{(t-1)}\right]\left(d x^{(t-1)}\right) .
\end{aligned}
$$

The first term in the RHS converges to zero from condition (ii). The second term in the RHS converges to zero since $\nu_{n}^{(t-1)}$ converges weakly to $\nu^{(t-1)}$ and the function mapping $x^{(t-1)}$ into $E\left[e^{i u_{t} \xi_{t}} \mid \xi_{t-1}=x_{t-1}\right] e^{i u_{1} x_{1}+\ldots+i u_{t-1} x_{t-1}}$ is bounded and continuous from condition (iii). The conclusion follows.

Let us check the conditions of Lemma A.1. Condition (i) is satisfied by assumption. Condition (iii) is satisfied since the conditional characteristic function of the limit Gaussian process is given by:

$$
E\left[\exp \left(-i u \xi_{t}\right) \mid \xi_{t-1}=x\right]=\exp \left(-i u \rho x-\frac{1}{2} u^{2} \eta^{2}\right),
$$

which is a bounded and continuous function of $x \in \mathbb{R}$. Finally, let us check condition (ii). From equation (2.4) and its extension to the complex domain, the conditional characteristic function of $X_{n, t}=\sqrt{n}\left(N_{t} / n-\mu\right)$ is such that:

$$
\begin{aligned}
& \log E_{t-1}\left[\exp \left(-i u X_{n, t}\right)\right] \\
= & N_{t-1} \log \left[p_{11} \exp (-i u / \sqrt{n})+1-p_{11}\right]+\left(n-N_{t-1}\right) \log \left[p_{10} \exp (-i u / \sqrt{n})+1-p_{10}\right] \\
& +\sqrt{n} i u \mu .
\end{aligned}
$$

By a Taylor expansion when $n \rightarrow \infty$, we get:

$$
\begin{aligned}
& \log \left[p_{11} \exp (-i u / \sqrt{n})+1-p_{11}\right]=\log \left[1-p_{11} i u / \sqrt{n}-p_{11} u^{2} /(2 n)+O\left(1 / n^{3 / 2}\right)\right] \\
= & -p_{11} i u / \sqrt{n}-p_{11}\left(1-p_{11}\right) u^{2} / 2 n+O\left(1 / n^{3 / 2}\right),
\end{aligned}
$$


and similarly:

$$
\log \left[p_{10} \exp (-i u / \sqrt{n})+1-p_{10}\right]=-p_{10} i u / \sqrt{n}-p_{10}\left(1-p_{10}\right) u^{2} / 2 n+O\left(1 / n^{3 / 2}\right) .
$$

Then, we get:

$$
\begin{aligned}
& \log E_{t-1}\left[\exp \left(-i u X_{n, t}\right)\right] \\
= & \left(\mu n+\sqrt{n} X_{n, t-1}\right)\left[-p_{11} i u / \sqrt{n}-p_{11}\left(1-p_{11}\right) u^{2} /(2 n)\right] \\
& +\left[(1-\mu) n-\sqrt{n} X_{n, t-1}\right]\left[-p_{10} i u / \sqrt{n}-p_{10}\left(1-p_{10}\right) u^{2} /(2 n)\right]+\sqrt{n} i u \mu+O(1 / \sqrt{n}) \\
= & i u \sqrt{n}\left[-p_{10}(1-\mu)-p_{11} \mu+\mu\right] \\
& -i u X_{n, t-1}\left(p_{11}-p_{10}\right)-\left(u^{2} / 2\right)\left[\mu p_{11}\left(1-p_{11}\right)+(1-\mu) p_{10}\left(1-p_{10}\right)\right] \\
& -\frac{u^{2}}{2 \sqrt{n}}\left[p_{11}\left(1-p_{11}\right)-p_{10}\left(1-p_{10}\right)\right] X_{n, t-1}+O(1 / \sqrt{n}),
\end{aligned}
$$

where the $O(1 / \sqrt{n})$ term is independent of $X_{n, t-1}$, since $N_{t-1} / n \leq 1$. The first term of the RHS is equal to 0 by definition of the long run parameter. The sum of the second and third terms is the $\log$-characteristic function of a Gaussian distribution with mean $\left(p_{11}-p_{10}\right) X_{n, t-1}=\rho X_{n, t-1}$, and variance $\mu p_{11}\left(1-p_{11}\right)+(1-\mu) p_{10}\left(1-p_{10}\right)=\mu(1-\mu)\left(1-\rho^{2}\right)=\eta^{2}$. Thus, we get:

$$
E\left[\exp \left(-i u X_{n, t}\right) \mid X_{n, t-1}=x\right]=E\left[\exp \left(-i u \xi_{t}\right) \mid \xi_{t-1}=x\right] \exp \left(-\frac{\kappa u^{2}}{2 \sqrt{n}} x\right)(1+O(1 / \sqrt{n})),
$$

where $\kappa=p_{11}\left(1-p_{11}\right)-p_{10}\left(1-p_{10}\right)$ and the $O(1 / \sqrt{n})$ term is uniform w.r.t. $x$. We deduce that:

$$
\left|E\left[e^{i u X_{n, t}} \mid X_{n, t-1}=x\right]-E\left[e^{i u \xi_{t}} \mid \xi_{t-1}=x\right]\right| \leq\left|e^{-\frac{\kappa u^{2}}{2 \sqrt{n}} x}(1+O(1 / \sqrt{n}))-1\right| .
$$

Now, since $X_{n, t-1} / \sqrt{n} \in[-\mu, 1-\mu]$ and the exponential function is such that $\left|e^{x}-1\right| \leq C_{1}|x|$, for $x$ in a bounded interval around 0 and some constant $C_{1}$, we get:

$$
\begin{aligned}
& \int\left|E\left[e^{i u X_{n, t}} \mid X_{n, t-1}=x\right]-E\left[e^{i u \xi_{t}} \mid \xi_{t-1}=x\right]\right| \nu_{n, t-1}(d x) \leq C_{2} \frac{1}{\sqrt{n}} E\left[\left|X_{n, t-1}\right|\right]+O(1 / \sqrt{n}) \\
& \leq C_{2}\left(\frac{1}{n} E\left[X_{n, t-1}^{2}\right]\right)^{1 / 2}+O(1 / \sqrt{n})=C_{2} E\left[\left(N_{t-1} / n-\mu\right)^{2}\right]^{1 / 2}+O(1 / \sqrt{n}),
\end{aligned}
$$

for a constant $C_{2}$ (that may depend on $u$ ). To bound the expectation in the RHS, we use $E\left[\left(N_{t-1} / n-\right.\right.$ $\left.\mu)^{2}\right]=E\left[V\left[N_{t-1} / n \mid N_{1}\right]\right]+V\left[E\left[N_{t-1} / n \mid N_{1}\right]\right]+\left(E\left[N_{t-1} / n\right]-\mu\right)^{2}$ and $N_{t-1} \sim \mathcal{B}\left(N_{1}, p_{11}^{(t-1)}\right) *$ $\mathcal{B}\left(n-N_{1}, p_{10}^{(t-1)}\right)$ conditional on $N_{1}$, where $p_{11}^{(t-1)}=\mu+\rho^{t-1}(1-\mu)$ and $p_{10}^{(t-1)}=\mu-\rho^{t-1} \mu$. We get $E\left[N_{t-1} / n \mid N_{1}\right]=\mu+\rho^{t-1}\left(N_{1} / n-\mu\right), V\left[N_{t-1} / n \mid N_{1}\right] \leq 1 /(2 n)$, and:

$E\left[\left(N_{t-1} / n-\mu\right)^{2}\right]=\rho^{2(t-1)} V\left[N_{1} / n\right]+\left(E\left[N_{1} / n\right]-\mu\right)^{2}+O(1 / n)=O\left(\frac{1}{n}\left(E\left[X_{n, 1}^{2}\right]+1\right)\right)$. 
Since $E\left[X_{n, 1}^{2}\right]=O(1)$ by the assumptions in Proposition 2, the RHS is $O(1 / n)$ and the conclusion follows.

\section{A.1.2 Proof of Corollary 1}

If $p_{10}$ is replaced by $p_{10 t}=p_{10}\left(N_{t-1} / n\right) \simeq p_{10}(\mu)+\frac{1}{\sqrt{n}} \frac{d p_{10}(\mu)}{d \mu} X_{n, t-1}$, we get the additional term $-i u X_{n, t-1}(1-\mu) \frac{d p_{10}(\mu)}{d \mu}+o(1)$ in the expansion (a.3). This provides the modification involved in Corollary 1.

\section{APPENDIX 2: Proof of Proposition 4}

\section{A.2.1 Existence of a unique invariant measure for Markov process $\left(X_{t}\right)$}

The Markov process $\left(X_{t}\right)$ with $X_{t}=\left(\mu_{t}, F_{t}^{\prime}\right)^{\prime}$ has state space $\mathcal{X}=[0,1] \times \mathbb{R}^{q}$ and is such that:

$$
X_{t}=\Psi\left(X_{t-1}, \varepsilon_{t}\right)
$$

where:

$$
\Psi(x, \varepsilon)=\left(\begin{array}{c}
p_{11} \mu+(1-\mu) p_{10}[a(f, \varepsilon), \mu] \\
a(f, \varepsilon)
\end{array}\right),
$$

and $x=\left(\mu, f^{\prime}\right)^{\prime} \in \mathcal{X}$. We first exploit the nonlinear autoregressive (NLAR) representation (a.4) to show that Markov process $\left(X_{t}\right)$ is irreducible. We refer to Meyn, Tweedie (MT, 2009) for definition and results used below.

\section{i) Irreducibility}

A Markov process $\left(X_{t}\right)$ with state space $\mathcal{X} \subset \mathbb{R}^{s}$ is $\lambda_{\Omega}$-irreducible, where $\lambda_{\Omega}$ is the Lebesgue measure restricted on $\Omega \subset \mathcal{X}$, if $P\left[\tau_{A}<\infty \mid X_{0}=x\right]>0$ for any $x \in \mathcal{X}$ and any Borel set $A \in \mathcal{B}(\mathcal{X})$ such that $\lambda(A \cap \Omega)>0$, where $\tau_{A}=\inf \left\{t \geq 1: X_{t} \in A\right\}$ denotes the first passage time to set $A$. Thus, a Markov process is $\lambda_{\Omega}$-irreducible if it can reach in a finite time any subset of the state space with non-zero Lebesgue measure restricted on $\Omega$, starting from any initial point. 
Let us focus on a Markov process $\left(X_{t}\right)$ with nonlinear autoregressive representation as in (a.4). Let us define by $A^{1}(x)=\left\{\Psi(x, \varepsilon): \varepsilon \in \mathbb{R}^{m}\right\}$, the set of states that can be accessed from $x \in \mathcal{X}$, and let $A^{1}(C)=\left\{A^{1}(x): x \in C\right\}$ for set $C \subset \mathcal{X}$. Then, we define recursively the set of accessible states at horizon $t$, for $t \geq 2$, by $A^{t}(x)=A^{1}\left(A^{t-1}(x)\right)$. The next result is similar to Propositions 7.2.4-7.2.6 in MT.

Lemma A.2: Let Markov process $\left(X_{t}\right)$ admit the nonlinear autoregressive representation (a.4) where $\left(\varepsilon_{t}\right)$ is a strong white noise with positive p.d.f. on the support $\mathbb{R}^{m}$ and function $\Psi$ is differentiable w.r.t. both arguments. Let us assume that:

(i) The set $A^{t}(x)$ has non-empty interior, for any $x \in \mathcal{X}$ and any $t \in \mathbb{N}$ sufficiently large.

(ii) The set $\Omega(x):=\bigcap_{\tau=1}^{\infty} \bigcup_{t=\tau}^{\infty} A^{t}(x)$ is independent of $x \in \mathcal{X}$, that is, $\Omega(x)=\Omega$, for any $x \in \mathcal{X}$ and some subset $\Omega \subset \mathcal{X}$ of the state space.

Then, the Markov process $\left(X_{t}\right)$ is $\lambda_{\Omega}$-irreducible.

The set $\Omega(x)$ in condition (ii) of Lemma A.2 is the set of states accessible by the process $\left(X_{t}\right)$ in the long run when starting from $X_{0}=x$. Thus, condition (ii) of Lemma A.2 requires that any state in set $\Omega$ is accessible in the long run independently of the starting value of the process.

Let us apply Lemma A.2 for Markov process $X_{t}=\left(\mu_{t}, F_{t}^{\prime}\right)^{\prime}$, for which function $\Psi$ in the NLAR representation is given by (a.5).

Proposition A.1: Under Assumptions A.4 (i)-(iii) the Markov process $X_{t}=\left(\mu_{t}, F_{t}^{\prime}\right)^{\prime}$ defined in equations (a.4)-(a.5) is $\lambda_{\Omega}$-irreducible, where:

$$
\Omega=\left\{\left(p_{11} \mu+(1-\mu) p_{10}(f, \mu), f^{\prime}\right)^{\prime}: \mu \in[0,1], f \in \mathbb{R}^{q}\right\}
$$

Proof of Proposition A.1: Let us derive the sets $A^{t}(x)$ for $x \in \mathcal{X}$ and $t \in \mathbb{N}$. Define $\psi(\mu, f)=$ $p_{11} \mu+(1-\mu) p_{10}(f, \mu)$. We have for $t=1$ :

$$
A^{1}(x)=\left\{\left(\psi(\mu, a(f, \varepsilon)), a(f, \varepsilon)^{\prime}\right)^{\prime}: \varepsilon \in \mathbb{R}^{m}\right\}=\left\{\left(\psi(\mu, \tilde{f}), \tilde{f}^{\prime}\right)^{\prime}: \tilde{f} \in \mathbb{R}^{q}\right\}, \quad x=\left(\mu, f^{\prime}\right)^{\prime}
$$

from Assumption A.4 (iii). Thus, set $A^{1}(x)$ depends on $x=\left(\mu, f^{\prime}\right)^{\prime}$ via $\mu$ only. To derive sets $A^{t}(x)$ for $t \geq 2$, it is useful to derive first the sets of accessible states for component $\mu_{t}$ of the process $X_{t}$. Define $A_{*}^{1}(\mu)=\left\{\psi(\mu, f): f \in \mathbb{R}^{q}\right\}$ for $\mu \in[0,1], A_{*}^{1}(C)=\left\{A_{*}^{1}(\mu): \mu \in C\right\}$ for 
$C \subset[0,1]$, and recursively $A_{*}^{t}(\mu)=A_{*}^{1}\left(A_{*}^{t-1}(\mu)\right)$, for $t \geq 2$. From Assumption A.4 (ii) we have $A_{*}^{1}(\mu)=\left(a_{1}(\mu), b_{1}(\mu)\right)$, where $a_{1}(\mu)=p_{11} \mu$ and $b_{1}(\mu)=1-\left(1-p_{11}\right) \mu$. Functions $a_{1}$ and $b_{1}$ are increasing, resp. decreasing, on $[0,1]$. By induction we deduce that $A_{*}^{t}(\mu)=\left(a_{t}(\mu), b_{t}(\mu)\right)$, where $a_{t}(\mu)=a_{1}\left(a_{t-1}(\mu)\right)=p_{11}^{t} \mu$ and $b_{t}(\mu)=b_{1}\left(a_{t-1}(\mu)\right)=1-\left(1-p_{11}\right) p_{11}^{t-1} \mu$, for $t \geq 2$. Thus, we get:

$$
\begin{aligned}
A^{t}(x) & =\left\{\left(\psi(\tilde{\mu}, \tilde{f}), \tilde{f}^{\prime}\right)^{\prime}: \tilde{\mu} \in A_{*}^{t-1}(\mu), \tilde{f} \in \mathbb{R}^{q}\right\} \\
& =\left\{\left(\psi(\tilde{\mu}, \tilde{f}), \tilde{f}^{\prime}\right)^{\prime}: \tilde{\mu} \in\left(p_{11}^{t-1} \mu, 1-\left(1-p_{11}\right) p_{11}^{t-2} \mu\right), \tilde{f} \in \mathbb{R}^{q}\right\}
\end{aligned}
$$

for $x=\left(\mu, f^{\prime}\right)^{\prime}$ and $t \geq 2$. From Assumption A.4 (i), conditions (i) and (ii) of Lemma A.2 are satisfied with $\Omega=\left\{\left(\psi(\mu, f), f^{\prime}\right)^{\prime}: \mu \in[0,1], f \in \mathbb{R}^{q}\right\}$ and the process $\left(X_{t}\right)$ is $\lambda_{\Omega}$-irreducible.

Since $P\left[X_{t} \in \Omega \mid X_{t-1}=x\right]=1$ for any $x \in \mathcal{X}$, the measure $\lambda_{\Omega}$ is the maximal irreducible measure of Markov process $X_{t}=\left(\mu_{t}, F_{t}^{\prime}\right)^{\prime}$ (see MT, Proposition 4.2.2 for the definition of maximal irreducible measure).

\section{ii) Feller property}

A Markov process $\left(X_{t}\right)$ with state space $\mathcal{X} \subset \mathbb{R}^{s}$ is a (weak) Feller chain if, for any bounded and continuous function $h$ on $\mathcal{X}$, the mapping $x \rightarrow E\left[h\left(X_{t}\right) \mid X_{t-1}=x\right]$ on $\mathcal{X}$ is bounded and continuous. Thus, a Markov process satisfies the Feller property if the associated conditional expectation operator maps bounded and continuous functions into bounded and continuous functions.

From Proposition 6.1.2 in MT, a Markov process $\left(X_{t}\right)$ with NLAR representation as in (a.4) is a Feller chain if the function $\Psi$ is differentiable w.r.t. both arguments. Thus, from Assumption A.4 (ii), it follows that the Markov process $X_{t}=\left(\mu_{t}, F_{t}^{\prime}\right)^{\prime}$ defined by equations (a.4)-(a.5) is a Feller chain.

\section{iii) Harris recurrence}

A $\lambda_{\Omega}$-irreducible Markov process $\left(X_{t}\right)$ with state space $\mathcal{X}$ is Harris recurrent if $P\left[\eta_{A}=\infty \mid X_{0}=x\right]=1$ for any $x \in A$ and any set $A \in \mathcal{B}(\mathcal{X})$ such that $\lambda(A \cap \Omega)>0$, where $\eta_{A}=\sum_{t=1}^{\infty} 1\left\{X_{t} \in A\right\}$ is the number of passages to set $A$. Hence a Markov process is recurrent if it returns an infinite number of times to any accessible subset of the state space, with probability 1. 
The next result follows directly from Theorems 6.0.1, 9.2.2 and 9.4.1 in MT.

Lemma A.3: Let $\left(X_{t}\right)$ be a $\lambda_{\Omega}$-irreducible Feller chain with state space $\mathcal{X} \subset \mathbb{R}^{s}$. Suppose that there exist a function $V: \mathcal{X} \rightarrow \mathbb{R}_{+}$and a compact set $C \subset \mathcal{X}$ such that:

$$
\Delta V(x):=E\left[V\left(X_{t}\right) \mid X_{t-1}=x\right]-V(x) \leq 0, \quad x \in C^{c},
$$

and $V(x) \rightarrow \infty$ if $x \in \mathcal{X}$ and $\|x\| \rightarrow \infty$. Then, the Markov process $\left(X_{t}\right)$ is Harris recurrent.

The inequality (a.7) is a drift condition and requires that a certain nonlinear transformation of the process reverts towards the center of the state space.

Let us now apply Lemma A.3 to the Markov process $X_{t}=\left(\mu_{t}, F_{t}^{\prime}\right)^{\prime}$ defined by equations (a.4)-(a.5).

Proposition A.2: Under Assumption A.4, the Markov process $X_{t}=\left(\mu_{t}, F_{t}^{\prime}\right)^{\prime}$ is Harris recurrent.

Proof of Proposition A.2: From Section ii), process $\left(X_{t}\right)$ is a $\lambda_{\Omega}$-irreducible Feller chain. Let us check the drift condition in (a.7). Consider the function $V(x)=\|f\|$, for $x=\left(\mu, f^{\prime}\right)^{\prime}$. Then, from Assumption A.4 (iv):

$$
\Delta V(x)=E\left[\left\|a\left(f, \varepsilon_{t}\right)\right\|\right]-\|f\| \leq c-(1-\gamma)\|f\| \leq 0
$$

for $\|f\| \geq \max \left\{R, \frac{c}{1-\gamma}\right\}=: R_{1}$. Then, the drift condition (a.7) follows with compact set $C=$ $[0,1] \times\left\{f \in \mathbb{R}^{q}:\|f\| \leq R_{1}\right\}$. Finally, we have $V(x) \rightarrow \infty$ if $\|f\| \rightarrow \infty$.

In the proof of Proposition A.2 we can use a function $V$ that is independent of $\mu$, since process $\left(\mu_{t}\right)$ is bounded.

\section{iv) Existence of a unique invariant probability measure and Law of Large Numbers (LLN)}

The next result follows from Theorems 10.4.9 and 17.1.7 in MT.

Lemma A.4: Let $\left(X_{t}\right)$ be a $\lambda_{\Omega}$-irreducible, Harris recurrent Markov process with state space $\mathcal{X} \subset \mathbb{R}^{s}$. Then, there exists a unique invariant probability measure $\pi$, which is equivalent to the Lebesgue measure $\lambda_{\Omega}$. Moreover, we have:

$$
\frac{1}{T} \sum_{t=1}^{T} h\left(X_{t}\right) \rightarrow \int_{\mathcal{X}} h(x) \pi(d x), \quad T \rightarrow \infty,
$$


$P$-a.s., for any function $h$ on $\mathcal{X}$ such that $\int_{\mathcal{X}} h(x) \pi(d x)<\infty$.

From Propositions A.1 and A.2, and Lemma A.4, we deduce:

Proposition A.3: Under Assumption A.4 the Markov process $X_{t}=\left(\mu_{t}, F_{t}^{\prime}\right)^{\prime}$ defined by equations (a.4)-(a.5) admits a unique invariant probability measure, which is equivalent to the Lebesgue measure $\lambda_{\Omega}$, where set $\Omega$ is defined in (a.6). Moreover, the LLN (a.9) holds for any function $h$ on $\mathcal{X}$ such that $\int_{\mathcal{X}} h(x) \pi(d x)<\infty$.

\section{A.2.2 Geometric ergodicity of Markov process $\left(X_{t}\right)$ [proof of Proposition 4} (i)]

\section{i) Aperiodicity}

A Markov process is aperiodic if there is no partition of the state space in two or more disjoint subsets through which the process cycles. More precisely, let $\left(X_{t}\right)$ be a $\lambda_{\Omega}$-irreducible Markov process with state space $\mathcal{X} \subset \mathbb{R}^{s}$. Then there exist an integer $d$ and disjoint sets $D_{1}, \ldots, D_{d} \in \mathcal{B}(\mathcal{X})$, called a $d$-cycle, such that (a) $x \in D_{i} \Rightarrow P\left[X_{t} \in D_{i+1} \mid X_{t-1}=x\right]=1$, for $i=1, \ldots, d(\bmod d)$,

and (b) $\lambda\left(\left(\bigcup_{i=1}^{d} D_{i}\right)^{c} \cap \Omega\right)=0$. The $d$-cycle is unique in the sense of Theorem 5.4.4 in MT, and the integer $d$ is a property of process $\left(X_{t}\right)$. If $d=1$, Markov process $\left(X_{t}\right)$ is aperiodic.

Let us now characterize aperiodicity for a Markov process $\left(X_{t}\right)$ with NLAR representation as in equation (a.4). From Proposition 7.2.5 in MT, under the conditions of Lemma A.2 the set $\Omega$ is the unique minimal set, that is, a closed and invariant set, which does not contain any closed and invariant set as a proper subset. Then, Propositions 7.2.5, 7.3.4 and 7.3.5 in MT allow to characterize aperiodicity in terms of connectedness of set $\Omega$.

Lemma A.5: Under the conditions of Lemma A.2, the Markov process $\left(X_{t}\right)$ is aperiodic if, and only if, the set $\Omega$ is connected.

We can apply this result for the Markov process $X_{t}=\left(\mu_{t}, F_{t}^{\prime}\right)^{\prime}$ defined by equations (a.4)-(a.5). From the proof of Proposition A.1, Assumptions A.4 (i)-(iii) imply the conditions of Lemma A.2. Moreover, set $\Omega$ defined in equation (a.6) is connected. Therefore, we deduce: 
Proposition A.4: Under Assumption A.4, the Markov process $X_{t}=\left(\mu_{t}, F_{t}^{\prime}\right)^{\prime}$ defined by equations (a.4)-(a.5) is aperiodic.

\section{ii) Geometric ergodicity}

For an irreducible, aperiodic Feller chain, geometric ergodicity can be characterized in terms of a drift condition. The next result corresponds to Theorem 15.0.1 in MT.

Lemma A.6: Let $\left(X_{t}\right)$ be a $\lambda_{\Omega}$-irreducible, aperiodic Feller chain with state space $\mathcal{X} \subset \mathbb{R}^{s}$. Suppose there exist a function $V$ on $\mathcal{X}$, with $V \geq 1$, a compact set $C \subset \mathcal{X}$ and constants $b<\infty$ and $\beta>0$ such that:

$$
\Delta V(x) \leq-\beta V(x)+b 1\{x \in C\}
$$

for any $x \in \mathcal{X}$. Then, the Markov process $\left(X_{t}\right)$ is $V$-geometrically ergodic.

We can apply this result for the Markov process $X_{t}=\left(\mu_{t}, F_{t}^{\prime}\right)^{\prime}$ defined by equations (a.4)-(a.5).

Proposition A.5: Under Assumption A.4, the Markov process $X_{t}=\left(\mu_{t}, F_{t}^{\prime}\right)^{\prime}$ is V-geometrically ergodic, where the function $V$ is defined by $V(x)=1+\|f\|$ for $x=\left(\mu, f^{\prime}\right)^{\prime}$.

Proof of Proposition A.5: From Propositions A.1-A.4, the Markov process $\left(X_{t}\right)$ is a $\lambda_{\Omega}$-irreducible, aperiodic Feller chain. Let $\beta \in(0,1-\gamma)$, where $\gamma$ is defined in Assumption A.4 (iv). Then, similarly as in inequality (a.8), from Assumption A.4 (iv) we have:

$$
\Delta V(x) \leq c-(1-\gamma)\|f\|=c+1-\gamma-(1-\gamma) V(x) \leq-\beta V(x)
$$

for any $x \in \mathcal{X}$ such that $\|f\| \geq \max \left\{R, \frac{c+1-\gamma}{1-\gamma-\beta}-1\right\}=: R_{2}$. Moreover, we have:

$$
\Delta V(x) \leq \sup _{f:\|f\| \leq R_{2}} E\left[\left\|a\left(f, \varepsilon_{t}\right)\right\|\right] \leq \sup _{f:\|f\| \leq R_{2}} E\left[\left\|a\left(f, \varepsilon_{t}\right)\right\|\right]+\beta\left(1+R_{2}\right)-\beta V(x)
$$

for any $x \in \mathcal{X}$ such that $\|f\| \leq R_{2}$, where $\sup _{f:\|f\| \leq R_{2}} E\left[\left\|a\left(f, \varepsilon_{t}\right)\right\|\right]<\infty$ from Assumption A.4 (iv). By combining inequalities (a.11) and (a.12), we get the drift condition (a.10) with compact set $C=[0,1] \times\left\{f \in \mathbb{R}^{q}:\|f\| \leq R_{2}\right\}$ and constant $b=\sup _{f:\|f\| \leq R_{2}} E\left[\left\|a\left(f, \varepsilon_{t}\right)\right\|\right]+\beta\left(1+R_{2}\right)$. 


\section{A.2.3 Strict stationarity of process $\left(\xi_{t}^{*}\right)$ [Proof of Proposition 4 (ii)]}

We use the next result for linear autoregressive processes with stochastic autoregressive coefficient due to Brandt (1986).

Lemma A.7: Let process $\left(\xi_{t}^{*}\right)$ be such that:

$$
\xi_{t}^{*}=\rho_{t}^{*} \xi_{t-1}^{*}+Z_{t}
$$

where process $\left(\rho_{t}^{*}, Z_{t}\right)$ is strictly stationary and ergodic, and:

$$
E\left[\log \left|\rho_{t}^{*}\right|\right]<0, \quad E\left[\max \left\{\log \left|Z_{t}\right|, 0\right\}\right]<\infty
$$

Then, equation (a.13) admits a strictly stationary solution $\left(\xi_{t}^{*}\right)$.

To prove Proposition 4 (ii), we apply Lemma A.7 with $Z_{t}=\eta_{t}^{*} \varepsilon_{t}^{*}$. From equations (3.7)-(3.8) we have that $\left(\rho_{t}^{*}, Z_{t}\right)$ is a function of $\left(\mu_{t-1}, F_{t}^{\prime}, \varepsilon_{t}^{*}\right)^{\prime}$. From Proposition A.3, if process $\left(\mu_{t}, F_{t}^{\prime}\right)^{\prime}$ is initialized with its invariant distribution, then $\left(\rho_{t}^{*}, Z_{t}\right)$ is strictly stationary and ergodic. Moreover, since $\eta_{t}^{* 2} \leq 1 / 2$, the second inequality in (a.14) is satisfied. Then, from Lemma A.7 we deduce that process $\left(\xi_{t}^{*}\right)$ is strictly stationary if inequality (3.11) holds.

\section{APPENDIX 3: Gaussian model with logistic contagion}

i) Let us first show that the solution $\mu=\mu\left(a, b, p_{11}\right) \in(0,1)$ of equation (3.13) exists and is unique. Define the function:

$$
\psi(\mu)=\frac{1 / \mu-1}{1+\exp (-a \mu-b)}, \quad \mu \in(0,1) .
$$

Since function $\psi$ is continuous on the interval $(0,1)$ and such that $\psi(0)=\infty, \psi(1)=0$, the equation $\psi(\mu)=1-p_{11}$ for $p_{11} \in(0,1)$ admits a solution $\mu \in(0,1)$. The solution is unique, if function $\psi$ is monotonically decreasing. The first-order derivative is given by:

$$
\begin{aligned}
\frac{d \psi(\mu)}{d \mu} & =\frac{-\frac{1}{\mu^{2}}\left(1+e^{-a \mu-b}\right)+(1 / \mu-1)(-a) e^{-a \mu-b}}{\left(1+e^{-a \mu-b}\right)^{2}} \\
& =-\frac{e^{-a \mu-b}}{\mu^{2}\left(1+e^{-a \mu-b}\right)^{2}}\left[e^{a \mu+b}-\left(a \mu^{2}-a \mu-1\right)\right] .
\end{aligned}
$$


Since $a \mu^{2}-a \mu-1<0$ for $\mu \in[0,1]$, we get $\frac{d \psi(\mu)}{d \mu}<0$ for $\mu \in(0,1)$, and the conclusion follows.

ii) Let us now study the dependence of equilibrium $\mu$ on parameters $a, b, p_{11}$. We make explicit the dependence of function $\psi$ on parameters $a$ and $b$ by writing $\psi(\mu)=\psi(\mu ; a, b)$. Then:

$$
\begin{aligned}
\frac{\partial \mu}{\partial a} & =-\frac{\partial \psi / \partial a}{\partial \psi / \partial \mu}=\mu \frac{\mu(1-\mu)}{e^{a \mu+b}-\left(a \mu^{2}-a \mu-1\right)}>0 \\
\frac{\partial \mu}{\partial b} & =-\frac{\partial \psi / \partial b}{\partial \psi / \partial \mu}=\frac{\mu(1-\mu)}{e^{a \mu+b}-\left(a \mu^{2}-a \mu-1\right)}>0 \\
\frac{\partial \mu}{\partial p_{11}} & =-\frac{1}{\partial \psi / \partial \mu}=\frac{\mu^{2}\left(1+e^{a \mu+b}\right)\left(1+e^{-a \mu-b}\right)}{e^{a \mu+b}-\left(a \mu^{2}-a \mu-1\right)}>0
\end{aligned}
$$

iii) Let us finally show that $\rho^{*}=\rho^{*}\left(a, b, p_{11}\right)$ is such that $\left|\rho^{*}\right|<1$. We have from Corollary 1 :

$$
\rho^{*}=p_{11}-p_{10}(\mu)+a(1-\mu) p_{10}(\mu)\left[1-p_{10}(\mu)\right],
$$

where $\mu=\mu\left(a, b, p_{11}\right)$ is the equilibrium value. Then $\rho^{*}>-p_{10}(\mu) \geq-1$. Moreover, by using that $\mu=\mu\left(a, b, p_{11}\right)$ solves equation (3.13), we get:

$$
\begin{array}{ll} 
& \rho^{*}=p_{11}-p_{10}(\mu)+a \mu\left(1-p_{11}\right)\left[1-p_{10}(\mu)\right]<1 \\
\Leftrightarrow & \left(1-p_{11}\right)\left\{a \mu\left[1-p_{10}(\mu)\right]-1\right\}<p_{10}(\mu) \\
\Leftrightarrow & a \mu\left[1-p_{10}(\mu)\right]-1<\frac{\mu}{1-\mu} \\
\Leftrightarrow & a \mu(1-\mu)\left[1-p_{10}(\mu)\right]<1 .
\end{array}
$$

Now, by using that $1-p_{10}(\mu)=e^{-a \mu-b} /\left(1+e^{-a \mu-b}\right)$ and $a \mu e^{-a \mu}<1$ for $a \mu \geq 0$, we deduce that the latter inequality in (a.15) is satisfied for any values of the parameters. 


\section{A.4.1 Proof of Proposition 5}

We have:

$$
\begin{aligned}
\psi_{1 t}(u, v)= & E\left[\exp \left(-u N_{t+1}-v F_{t+1}\right) \underline{\mid N_{t}}, \underline{F_{t}}\right] \\
= & E\left\{E\left[\exp \left(-u N_{t+1}-v F_{t+1}\right) \underline{\mid N_{t}}, \underline{F_{t+1}}\right] \mid \underline{N_{t}}, \underline{F_{t}}\right\} \\
= & E\left[\operatorname { e x p } ( - v F _ { t + 1 } ) \operatorname { e x p } \left\{-\left(c_{0}+c_{1} F_{t+1}+c_{2} N_{t}\right)[1-\exp (-u)]\right.\right. \\
& \left.\left.+N_{t} \log [1-\rho+\rho \exp (-u)]\right\} \underline{\mid N_{t}}, \underline{F_{t}}\right]
\end{aligned}
$$

by applying equation (2.6) and Assumption A.5. Therefore, from Assumption A.6 we get:

$$
\begin{aligned}
\psi_{1 t}(u, v)= & \exp \left\{-c_{0}[1-\exp (-u)]-N_{t}\left(c_{2}[1-\exp (-u)]-\log [1-\rho+\rho \exp (-u)]\right)\right\} \\
& E\left[\exp \left\{-F_{t+1}\left(v+c_{1}[1-\exp (-u)]\right)\right\} \mid F_{t}\right] \\
= & \exp \left\{-c_{0}[1-\exp (-u)]-\beta\left(v+c_{1}[1-\exp (-u)]\right)\right. \\
& -N_{t}\left(c_{2}[1-\exp (-u)]-\log [1-\rho+\rho \exp (-u)]\right) \\
& \left.-F_{t} \alpha\left(v+c_{1}[1-\exp (-u)]\right)\right\} .
\end{aligned}
$$

\section{A.4.2 Proof of Proposition 7}

We use the following Lemma, which is proved at the end of this Appendix.

Lemma A.8: The conditional moments of order 1 and 2 of the joint process $\left(N_{t}, F_{t}\right)$ are given by:

$$
E_{t}\left(\begin{array}{c}
N_{t+1} \\
F_{t+1}
\end{array}\right)=\left(\begin{array}{c}
c_{0}+\delta \eta c_{1} \\
\delta \eta
\end{array}\right)+\left(\begin{array}{cc}
c_{2}+\rho & \gamma c_{1} \\
0 & \gamma
\end{array}\right)\left(\begin{array}{c}
N_{t} \\
F_{t}
\end{array}\right)
$$

and:

$V_{t}\left(\begin{array}{c}N_{t+1} \\ F_{t+1}\end{array}\right)=\left(\begin{array}{cc}c_{0}+\delta \eta c_{1}\left(1+\eta c_{1}\right)+\left[c_{2}+\rho(1-\rho)\right] N_{t}+\left(c_{1} \gamma+2 c_{1}^{2} \eta \gamma\right) F_{t} & \delta \eta^{2} c_{1}+2 \eta \gamma c_{1} F_{t} \\ \delta \eta^{2} c_{1}+2 \eta \gamma c_{1} F_{t} & \delta \eta^{2}+2 \eta \gamma F_{t}\end{array}\right)$

where $E_{t}$ and $V_{t}$ denote conditional expectation and variance given the past $\left(\underline{N_{t}}, \underline{F_{t}}\right)$ of the joint process. 
The conditional means, variances and covariances are linear functions of $N_{t}$ and $F_{t}$ since the joint process $\left(N_{t}, F_{t}\right)$ is $\mathrm{CaR}(1)$ (see Proposition 5).

Let us now prove Proposition 7. The unconditional moments of process $\left(N_{t}, F_{t}\right)$ are derived from the conditional moments in Lemma A.8 by applying the Law of Iterated Expectation and the variance decomposition formula. Specifically, from Lemma A.8 and the Law of Iterated Expectation we get:

$$
E\left[F_{t+1}\right]=E\left[E_{t}\left(F_{t+1}\right)\right]=\delta \eta+\gamma E\left[F_{t}\right]
$$

By stationarity we have $E\left[F_{t+1}\right]=E\left[F_{t}\right]$, and we get:

$$
E\left[F_{t}\right]=\frac{\delta \eta}{1-\gamma}
$$

[see also Gourieroux, Jasiak (2006) for the unconditional moments of the ARG process]. When $\eta=(1-\gamma) / \delta$, we get $E\left[F_{t}\right]=1$. Then, from Lemma A.8 and $\delta \eta=1-\gamma$ :

$E\left[N_{t+1}\right]=E\left[E_{t}\left[N_{t+1}\right]\right]=c_{0}+\delta \eta c_{1}+E\left[N_{t}\right]\left(c_{2}+\rho\right)+\gamma c_{1} E\left[F_{t}\right]=c_{0}+c_{1}+E\left[N_{t}\right]\left(c_{2}+\rho\right)$,

which yields:

$$
E\left[N_{t}\right]=\frac{c_{0}+c_{1}}{1-\left(c_{2}+\rho\right)}
$$

Let us now consider the unconditional variance of $F_{t}$. From Lemma A.8 and the variance decomposition formula, we get:

$$
\begin{aligned}
V\left[F_{t+1}\right] & =V\left[E_{t}\left(F_{t+1}\right)\right]+E\left[V_{t}\left(F_{t}\right)\right]=V\left[\delta \eta+\gamma F_{t}\right]+E\left[\delta \eta^{2}+2 \eta \gamma F_{t}\right] \\
& =\gamma^{2} V\left[F_{t}\right]+\delta \eta^{2}+2 \frac{\delta \eta^{2} \gamma}{1-\gamma}=\gamma^{2} V\left[F_{t}\right]+\delta \eta^{2} \frac{1+\gamma}{1-\gamma}
\end{aligned}
$$

which yields:

$$
V\left[F_{t}\right]=\frac{1}{1-\gamma^{2}} \delta \eta^{2} \frac{1+\gamma}{1-\gamma}=\frac{\delta \eta^{2}}{(1-\gamma)^{2}}=\frac{1}{\delta}
$$

Let us now consider the unconditional covariance between $F_{t}$ and $N_{t}$. We have:

$$
\begin{aligned}
\operatorname{Cov}\left(F_{t+1}, N_{t+1}\right) & =E\left[\operatorname{Cov}_{t}\left(F_{t+1}, N_{t+1}\right)\right]+\operatorname{Cov}\left(E_{t}\left[F_{t+1}\right], E_{t}\left[N_{t+1}\right]\right) \\
& =E\left[\delta \eta^{2} c_{1}+2 \eta \gamma c_{1} F_{t}\right]+\operatorname{Cov}\left(\delta \eta+\gamma F_{t}, c_{0}+\delta \eta c_{1}+N_{t}\left(c_{2}+\rho\right)+\gamma c_{1} F_{t}\right) \\
& =\delta \eta^{2} c_{1} \frac{1+\gamma}{1-\gamma}+\gamma^{2} c_{1} V\left[F_{t}\right]+\gamma\left(c_{2}+\rho\right) \operatorname{Cov}\left(F_{t}, N_{t}\right) \\
& =\frac{c_{1}}{\delta}+\gamma\left(c_{2}+\rho\right) \operatorname{Cov}\left(F_{t}, N_{t}\right)
\end{aligned}
$$


which yields:

$$
\operatorname{Cov}\left(F_{t}, N_{t}\right)=\frac{c_{1}}{\delta} \frac{1}{1-\gamma\left(c_{2}+\rho\right)}
$$

Finally, let us consider the unconditional variance of $N_{t}$. We have:

$$
\begin{aligned}
V\left[N_{t+1}\right]= & E\left[V_{t}\left(N_{t+1}\right)\right]+V\left[E_{t}\left(N_{t+1}\right)\right] \\
= & E\left[c_{0}+\delta \eta c_{1}\left(1+\eta c_{1}\right)+N_{t}\left(c_{2}+\rho(1-\rho)\right)+F_{t}\left(c_{1} \gamma+2 c_{1}^{2} \eta \gamma\right)\right] \\
& +V\left[c_{0}+\delta \eta c_{1}+N_{t}\left(c_{2}+\rho\right)+\gamma c_{1} F_{t}\right] \\
= & c_{0}+\delta \eta c_{1}\left(1+\eta c_{1}\right)+\frac{c_{2}+\rho(1-\rho)}{1-\left(c_{2}+\rho\right)}\left(c_{0}+c_{1}\right)+c_{1} \gamma+2 c_{1}^{2} \eta \gamma \\
& +\left(c_{2}+\rho\right)^{2} V\left[N_{t}\right]+\gamma^{2} c_{1}^{2} V\left[F_{t}\right]+2\left(c_{2}+\rho\right) c_{1} \gamma \operatorname{Cov}\left(N_{t}, F_{t}\right) \\
= & c_{0}+\delta \eta c_{1}\left(1+\eta c_{1}\right)+\frac{c_{2}+\rho(1-\rho)}{1-\left(c_{2}+\rho\right)}\left(c_{0}+c_{1}\right)+c_{1} \gamma+2 c_{1}^{2} \eta \gamma \\
& +\left(c_{2}+\rho\right)^{2} V\left[N_{t}\right]+\frac{\gamma c_{1}^{2}}{\delta}\left[\gamma+\frac{2\left(c_{2}+\rho\right)}{1-\gamma\left(c_{2}+\rho\right)}\right] .
\end{aligned}
$$

We deduce:

$$
\begin{aligned}
V\left[N_{t}\right]= & \frac{1}{1-\left(c_{2}+\rho\right)^{2}}\left\{c_{0}+\delta \eta c_{1}\left(1+\eta c_{1}\right)+\frac{c_{2}+\rho(1-\rho)}{1-\left(c_{2}+\rho\right)}\left(c_{0}+c_{1}\right)+c_{1} \gamma+2 c_{1}^{2} \eta \gamma\right. \\
& \left.+\frac{\gamma c_{1}^{2}}{\delta}\left[\gamma+\frac{2\left(c_{2}+\rho\right)}{1-\gamma\left(c_{2}+\rho\right)}\right]\right\} .
\end{aligned}
$$

By using $\eta=(1-\gamma) / \delta$ and rearranging terms, we get the formula for $V\left[N_{t}\right]$ given in Proposition 7.

\section{A.4.3 Proof of Proposition 8}

Let $Z_{t}=\left(N_{t}, F_{t}\right)^{\prime}$. Then, from Lemma A.8 we get $\operatorname{Cov}\left(Z_{t+h}, Z_{t}\right)=A^{h} V\left(Z_{t}\right)$, for $h \geq 0$, where the matrix $A$ is given by :

$$
A=\left(\begin{array}{cc}
c_{2}+\rho & \gamma c_{1} \\
0 & \gamma
\end{array}\right)
$$

Now, for a generic triangular $(2,2)$ matrix we have:

$$
\left(\begin{array}{ll}
a & b \\
0 & c
\end{array}\right)^{h}=\left(\begin{array}{cc}
a^{h} & b\left(a^{h-1}+c a^{h-2}+\ldots+c^{h-2} a+c^{h-1}\right) \\
0 & c^{h}
\end{array}\right) .
$$


Thus, we get:

$$
A^{h}=\left(\begin{array}{cc}
\left(c_{2}+\rho\right)^{h} & b(h) \\
0 & \gamma^{h}
\end{array}\right)
$$

where:

$$
b(h)=\gamma c_{1}\left[\left(c_{2}+\rho\right)^{h-1}+\gamma\left(c_{2}+\rho\right)^{h-2}+\ldots+\gamma^{h-2}\left(c_{2}+\rho\right)+\gamma^{h-1}\right]
$$

When $\gamma=0$, we have $b(h)=0$, which implies $\operatorname{Cov}\left(N_{t+h}, N_{t}\right)=\left(c_{2}+\rho\right)^{h} V\left(N_{t}\right)$ and thus $\operatorname{Corr}\left(N_{t+h}, N_{t}\right)=\left(c_{2}+\rho\right)^{h}$. Let us now consider the case $\gamma>0$. Then:

$$
\begin{aligned}
b(h) & =c_{1} \gamma^{h}\left[1+\frac{c_{2}+\rho}{\gamma}+\left(\frac{c_{2}+\rho}{\gamma}\right)^{2}+\ldots+\left(\frac{c_{2}+\rho}{\gamma}\right)^{h-1}\right] \\
& =c_{1} \gamma^{h} \frac{1-\left(\frac{c_{2}+\rho}{\gamma}\right)^{h}}{1-\frac{c_{2}+\rho}{\gamma}}=c_{1} \gamma \frac{\gamma^{h}-\left(c_{2}+\rho\right)^{h}}{\gamma-\left(c_{2}+\rho\right)}
\end{aligned}
$$

if $\gamma \neq c_{2}+\rho$, and:

$$
b(h)=c_{1} h \gamma^{h}
$$

if $\gamma=c_{2}+\rho$. It follows:

$$
\operatorname{Cov}\left(N_{t+h}, N_{t}\right)=\left(c_{2}+\rho\right)^{h} V\left(N_{t}\right)+b(h) \operatorname{Cov}\left(N_{t}, F_{t}\right)
$$

and:

$$
\operatorname{Corr}\left(N_{t+h}, N_{t}\right)=\left(c_{2}+\rho\right)^{h}+b(h) \frac{\operatorname{Cov}\left(N_{t}, F_{t}\right)}{V\left(N_{t}\right)} .
$$

The conclusion follows.

\section{A.4.4 Conditional dispersion}

From the Law of Iterated Expectation and the variance decomposition formula we have:

$$
\begin{aligned}
E\left[N_{t+1} \mid N_{t}\right] & =E\left[E\left(N_{t+1} \mid N_{t}, F_{t}\right) \mid N_{t}\right] \\
V\left[N_{t+1} \mid N_{t}\right] & =E\left[V\left(N_{t+1} \mid N_{t}, F_{t}\right) \mid N_{t}\right]+V\left[E\left(N_{t+1} \mid N_{t}, F_{t}\right) \mid N_{t}\right] .
\end{aligned}
$$

From Lemma A.8, we get:

$$
E\left[N_{t+1} \mid N_{t}\right]=c_{0}+\delta \eta c_{1}+\left(c_{2}+\rho\right) N_{t}+\gamma c_{1} E\left[F_{t} \mid N_{t}\right]
$$


and:

$V\left[N_{t+1} \mid N_{t}\right]=c_{0}+\delta \eta c_{1}\left(1+\eta c_{1}\right)+\left[c_{2}+\rho(1-\rho)\right] N_{t}+\left(c_{1} \gamma+2 c_{1}^{2} \eta \gamma\right) E\left[F_{t} \mid N_{t}\right]+\gamma^{2} c_{1}^{2} V\left[F_{t} \mid N_{t}\right]$

By using $\eta=(1-\gamma) / \delta$, equation (4.1) follows.

\section{A.4.5 Proof of Lemma A.8}

The conditional moments are derived from the expansion of the log conditional Laplace transform in Proposition 5 around $u=v=0$. At second order in $u, v$, we have:

$$
\begin{aligned}
\log \psi_{1 t}(u, v)= & -c_{0}[1-\exp (-u)]-\beta\left(v+c_{1}[1-\exp (-u)]\right) \\
& -N_{t}\left(c_{2}[1-\exp (-u)]-\log [1-\rho+\rho \exp (-u)]\right)-F_{t} \alpha\left(v+c_{1}[1-\exp (-u)]\right) \\
\simeq & -c_{0}\left(u-u^{2} / 2\right)-\beta\left(v+c_{1}\left[u-u^{2} / 2\right]\right) \\
& -N_{t}\left(c_{2}\left[u-u^{2} / 2\right]-\log \left[1-\rho u+\rho u^{2} / 2\right]\right)-F_{t} \alpha\left(v+c_{1}\left[u-u^{2} / 2\right]\right) \\
\simeq & -c_{0}\left(u-u^{2} / 2\right)-\beta\left(v+c_{1}\left[u-u^{2} / 2\right]\right) \\
& -N_{t}\left(c_{2}\left[u-u^{2} / 2\right]+\rho u-\rho(1-\rho) u^{2} / 2\right)-F_{t} \alpha\left(v+c_{1}\left[u-u^{2} / 2\right]\right) .
\end{aligned}
$$

By using the expansions at second order of functions $\alpha$ and $\beta$ :

$$
\alpha(u)=\frac{\gamma u}{1+\eta u} \simeq \gamma u-\gamma \eta u^{2}, \quad \beta(u)=\delta \log (1+\eta u) \simeq \delta \eta u-\frac{1}{2} \delta \eta^{2} u^{2},
$$

we get:

$$
\begin{aligned}
\alpha\left(v+c_{1}\left[u-u^{2} / 2\right]\right) & \simeq \gamma\left(v+c_{1} u-c_{1} u^{2} / 2\right)-\gamma \eta\left(v+c_{1} u\right)^{2} \\
\beta\left(v+c_{1}\left[u-u^{2} / 2\right]\right) & \simeq \delta \eta\left(v+c_{1} u-c_{1} u^{2} / 2\right)-\frac{1}{2} \delta \eta^{2}\left(v+c_{1} u\right)^{2}
\end{aligned}
$$

By replacing expansions (a.20) into expansion (a.19), and gathering the terms proportional to $u, v$, $u^{2}, v^{2}$ and $u v$, we get:

$$
\begin{aligned}
\log \psi_{1 t}(u, v) \simeq \quad & -\left[c_{0}+\delta \eta c_{1}+N_{t}\left(c_{2}+\rho\right)+\gamma c_{1} F_{t}\right] u-\left[\delta \eta+\gamma F_{t}\right] v \\
+ & \frac{1}{2}\left[c_{0}+\delta \eta c_{1}\left(1+\eta c_{1}\right)+N_{t}\left(c_{2}+\rho(1-\rho)\right)+F_{t}\left(c_{1} \gamma+2 c_{1}^{2} \eta \gamma\right)\right] u^{2} \\
+ & \frac{1}{2}\left[\delta \eta^{2}+2 \eta \gamma F_{t}\right] v^{2}+\left[\delta \eta^{2} c_{1}+2 F_{t} \eta \gamma c_{1}\right] u v .
\end{aligned}
$$

The conclusion follows. 